\title{
High-Order Algorithms for Riesz Derivative and Their Applications $(I)$
}

\author{
Hengfei Ding, ${ }^{1}$ Changpin Li, ${ }^{1}$ and YangQuan Chen ${ }^{2}$ \\ ${ }^{1}$ Department of Mathematics, Shanghai University, Shanghai 200444, China \\ ${ }^{2}$ School of Engineering, University of California, Merced, CA 95343, USA \\ Correspondence should be addressed to Changpin Li; lcp@shu.edu.cn
}

Received 24 March 2014; Accepted 22 April 2014; Published 22 May 2014

Academic Editor: Luisa Morgado

Copyright (c) 2014 Hengfei Ding et al. This is an open access article distributed under the Creative Commons Attribution License, which permits unrestricted use, distribution, and reproduction in any medium, provided the original work is properly cited.

\begin{abstract}
We firstly develop the high-order numerical algorithms for the left and right Riemann-Liouville derivatives. Using these derived schemes, we can get high-order algorithms for the Riesz fractional derivative. Based on the approximate algorithm, we construct the numerical scheme for the space Riesz fractional diffusion equation, where a fourth-order scheme is proposed for the spacial Riesz derivative, and where a compact difference scheme is applied to approximating the first-order time derivative. It is shown that the difference scheme is unconditionally stable and convergent. Finally, numerical examples are provided which are in line with the theoretical analysis.
\end{abstract}

\section{Introduction}

In recent decades, fractional differential equations are undergoing rapid development due to their wide application in physics, engineering, economics, and some other research realms [1-3]. Several methods have been introduced for some special fractional differential equations to seek their analytical solutions, such as the integral transformation method (Laplace's transform, Fourier's transform, and Mellin's transform), Adomian decomposition method, the method of separating variables, and so on [4-7]. However, the exact solutions of most fractional differential equations cannot be obtained. So it becomes important to develop numerical methods for these equations. It is worth mentioning that the fractional linear multistep (also high order) methods for the Riemann-Liouville integrals and derivatives were firstly proposed in [8]. High-order numerical methods for Caputo derivatives were firstly constructed in [9]. Other fractional differential equations include Caputo or (and) RiemannLiouville derivatives; there also existed some numerical approaches [10-13]. So, in this paper, our main aim is to construct high-order numerical methods for Riesz fractional derivative and Riesz fractional differential equations.
The Riesz fractional derivative was derived from the kinetics of chaotic dynamics [14, 15]. For the Riesz fractional differential equations, there have existed several analytical and numerical methods. Zhang and Liu [16] studied the analytical solutions of space Riesz and time Caputo fractional partial differential equations. Later on, they [17] used Galerkin finite element approximation and a backward difference technique in Riesz fractional advection-dispersion equation. By using the Laplace and Fourier transform, Chen et al. [18] obtained the analytical solution for the space Riesz fractional reaction dispersion equation, and they also constructed an explicit finite difference scheme for it. Shen et al. [19] presented the explicit and implicit difference schemes for the Riesz fractional advection-dispersion equation and the space Riesz time Caputo fractional advection-dispersion equation. Recently, they [20] proposed a novel numerical method for the space Riesz fractional advection-dispersion equation based on fractional central difference operator. Özdemir et al. [21] were concerned with the numerical solutions of a two-dimensional problem. Yang et al. [22] obtained some numerical solutions for the space Riesz fractional diffusion and advection-dispersion equations on a finite domain by using three numerical methods. Çelik and Duman [23] 
numerically approximated the fractional diffusion equation with the space Riesz fractional derivative on a finite domain according to the fractional central difference operator. Very recently, Wang et al. [24] consider the coupled nonlinear Schrödinger equations by using similar methods as in [23]. In the above mentioned numerical methods, the accuracy of the numerical methods for the Riesz fractional derivative is not more than 2; this motivates us to investigative high-order numerical schemes for it.

In the present paper, we study the following space Riesz fractional diffusion equation:

$$
\begin{array}{r}
\frac{\partial u(x, t)}{\partial t}=K \frac{\partial^{\alpha} u(x, t)}{\partial|x|^{\alpha}}+f(x, t), \\
a<x<b, \quad 0<t \leq T,
\end{array}
$$

with the corresponding initial and boundary value conditions:

$$
\begin{gathered}
u(x, 0)=\psi(x), \quad a \leq x \leq b, \\
\left.\mathrm{RL}_{a, x}^{\alpha-2} u(x, t)\right|_{x=a}=\phi(t), \quad 0 \leq t \leq T, \\
\left.\mathrm{RL}_{x, b}^{\alpha-2} u(x, t)\right|_{x=b}=\varphi(t), \quad 0 \leq t \leq T,
\end{gathered}
$$

where $K>0$ is a diffusion coefficient and $f(x, t), \phi(t), \varphi(t)$, and $\psi(x)$ are sufficiently smooth functions. $\partial^{\alpha} / \partial|x|^{\alpha}$ is the Riesz fractional derivative with order $\alpha(1<\alpha<2)$ defined as follows [22]:

$$
\frac{\partial^{\alpha} u(x, t)}{\partial|x|^{\alpha}}=-\Psi_{\alpha}\left({ }_{\mathrm{RL}} D_{a, x}^{\alpha}{ }_{\mathrm{RL}} D_{x, b}^{\alpha}\right) u(x, t),
$$

where $\Psi_{\alpha}=(1 / 2) \sec (\pi \alpha / 2)$ and ${ }_{\mathrm{RL}} D_{a, x}^{\alpha}$ and ${ }_{\mathrm{RL}} D_{x, b}^{\alpha}$ are the left- and right-side Riemann-Liouville fractional derivatives represented in the following form:

$$
\begin{array}{r}
{ }_{\mathrm{RL}} D_{a, x}^{\alpha} u(x, t)=\frac{1}{\Gamma(2-\alpha)} \frac{\partial^{2}}{\partial x^{2}} \int_{a}^{x} \frac{u(\xi, t)}{(x-\xi)^{\alpha-1}} d \xi, \\
x \in[a, b], \\
\mathrm{RL}_{x, b}^{\alpha} u(x, t)=\frac{1}{\Gamma(2-\alpha)} \frac{\partial^{2}}{\partial x^{2}} \int_{x}^{b} \frac{u(\xi, t)}{(\xi-x)^{\alpha-1}} d \xi, \\
x \in[a, b] ;
\end{array}
$$

${ }_{\mathrm{RL}} D_{a, x}^{\alpha-2}$ and ${ }_{\mathrm{RL}} D_{x, b}^{\alpha-2}$ are left- and right-side Riemann-Liouville fractional integral operators and defined by $(1<\alpha<2)$ :

$$
\begin{aligned}
& \mathrm{RL}_{a, x} D_{a-2}^{\alpha-2}(x, t)=\frac{1}{\Gamma(2-\alpha)} \int_{a}^{x}(x-\xi)^{1-\alpha} u(\xi, t) d \xi \\
& { }_{\mathrm{RL}} D_{x, b}^{\alpha-2} u(x, t)=\frac{1}{\Gamma(2-\alpha)} \int_{x}^{b}(\xi-x)^{1-\alpha} u(\xi, t) d \xi,
\end{aligned}
$$

in which $\Gamma(\cdot)$ indicates Euler's Gamma function.

The structure of the paper is outlined as follows. In Section 2, we list existing numerical approximation of the Riesz fractional derivative just for reference. Then, we focus on constructing one second-order scheme and two fourthorder numerical schemes. In Section 3, we only present one fourth-order numerical algorithm for the space Riesz fractional diffusion equation on a finite domain. In Sections 4 and 5 , we discuss the stability and convergence of the difference scheme, respectively. In Section 6, some numerical examples are shown which support the theoretical results derived in the above sections. Finally, the last section concludes this paper.

\section{The Numerical Approximation of the Riesz Derivative}

Firstly, we take the mesh points $x_{m}=a+m h, m=0,1, \ldots, M$, and $t_{n}=n \tau, n=0,1, \ldots, N$, where $h=(b-a) / M$ and $\tau=T / N$; that is, $h$ and $\tau$ are the uniform spatial stepsize and temporal stepsize.

As we all know, for every $\alpha(1<\alpha<2)$ the left and right Riemann-Liouville derivatives exist and coincide with the left and right Grünwald-Letnikov derivatives under suitable conditions, respectively, where the left and right GrünwaldLetnikov derivative definitions with order $\alpha$ are given below [6]:

$$
\begin{aligned}
\mathrm{GL}_{a, x} & D_{a}^{\alpha} u\left(x_{m}, t\right)=\frac{1}{h^{\alpha}} \sum_{k=0}^{m} \omega_{k}^{(\alpha)} u\left(x_{m-k}, t\right)+\mathcal{O}(h), \\
\mathrm{GL} & D_{x, b}^{\alpha} u\left(x_{m}, t\right)=\frac{1}{h^{\alpha}} \sum_{k=0}^{M-m} \omega_{k}^{(\alpha)} u\left(x_{m+k}, t\right)+\mathcal{O}(h),
\end{aligned}
$$

in which ${\omega_{k}^{(\alpha)}}_{k}=(-1)^{k}\left(\begin{array}{l}\alpha \\ k\end{array}\right)=\left((-1)^{k} \Gamma(1+\alpha)\right) /(\Gamma(1+k) \Gamma(1+$ $\alpha-k)$ ).

So, the Riesz derivative with order $\alpha \in(1,2)$ can be discretized in the following ways.

(I) By the Standard Grünwald-Letnikov Formula. Based on the above assumption and (3), we can obtain the first-order approximation formula

$$
\begin{aligned}
\frac{\partial^{\alpha} u\left(x_{m}, t\right)}{\partial|x|^{\alpha}}=-\frac{\Psi_{\alpha}}{h^{\alpha}}( & \sum_{k=0}^{m} \omega_{k}^{(\alpha)} u\left(x_{m-k}, t\right) \\
& \left.+\sum_{k=0}^{M-m} \Phi_{k}^{(\alpha)} u\left(x_{m+k}, t\right)\right)+\mathcal{O}(h) .
\end{aligned}
$$

(II) By the Shifted Grünwald-Letnikov Formula. In [25], Meerschaert and Tadjeran show that the above standard Grünwald-Letnikov formula is often unstable for time dependent problems. Hence, they proposed the following shifted Grünwald-Letnikov formulas for the left and right RiemannLiouville derivatives in order to overcome the instability:

$$
\begin{gathered}
\mathrm{RL}_{a, x}^{\alpha} u\left(x_{m}, t\right)=\sum_{k=0}^{m+1} \omega_{k}^{(\alpha)} u\left(x_{m-k+1}, t\right)+\mathcal{O}(h), \\
\mathrm{RL}_{x, b}^{\alpha} u\left(x_{m}, t\right)=\sum_{k=0}^{M-m+1} \omega_{k}^{(\alpha)} u\left(x_{m+k-1}, t\right)+\mathcal{O}(h) .
\end{gathered}
$$


Therefore, the modified first-order approximation scheme is constructed as follows:

$$
\begin{aligned}
\frac{\partial^{\alpha} u\left(x_{m}, t\right)}{\partial|x|^{\alpha}}=-\frac{\Psi_{\alpha}}{h^{\alpha}}( & \sum_{k=0}^{m+1} \omega_{k}^{(\alpha)} u\left(x_{m-k+1}, t\right) \\
& \left.+\sum_{k=0}^{M-m+1} \omega_{k}^{(\alpha)} u\left(x_{m+k-1}, t\right)\right)+\mathcal{O}(h) .
\end{aligned}
$$

(III) By the L2 Approximation Method. Note that the left and right Riemann-Liouville derivatives can be rewritten as $(1<$ $\alpha<2)$ :

$$
\begin{aligned}
{ }_{\mathrm{RL}} D_{a, x}^{\alpha} u(x, t)=\sum_{k=0}^{1} & \frac{x^{k-\alpha}}{\Gamma(k+1-\alpha)} \frac{\partial^{k} u(a, t)}{\partial x^{k}} \\
& +\frac{1}{\Gamma(2-\alpha)} \int_{a}^{x} \frac{\partial^{2} u(\xi, t)}{\partial \xi^{2}}(x-\xi)^{1-\alpha} d \xi, \\
{ }_{\mathrm{RL}} D_{x, b}^{\alpha} u(x, t)=\sum_{k=0}^{1} & \frac{(b-x)^{k-\alpha}}{\Gamma(k+1-\alpha)} \frac{\partial^{k} u(b, t)}{\partial x^{k}} \\
& +\frac{1}{\Gamma(2-\alpha)} \int_{x}^{b} \frac{\partial^{2} u(\xi, t)}{\partial \xi^{2}}(\xi-x)^{1-\alpha} d \xi .
\end{aligned}
$$

Hence, we can obtain a first-order scheme for the left and right Riemann-Liouville fractional derivatives [22]:

$$
\begin{aligned}
& { }_{\mathrm{RL}} D_{a, x}^{\alpha} u\left(x_{m}, t\right) \\
& =\frac{1}{\Gamma(3-\alpha) h^{\alpha}}\left\{\frac{(1-\alpha)(2-\alpha) u\left(x_{0}, t\right)}{m^{\alpha}}\right. \\
& +\frac{(2-\alpha)\left[u\left(x_{1}, t\right)-u\left(x_{0}, t\right)\right]}{m^{\alpha-1}} \\
& +\sum_{k=0}^{m-1} d_{k}^{(\alpha)}\left[u\left(x_{m-k+1}, t\right)\right. \\
& -2 u\left(x_{m-k}, t\right) \\
& \left.\left.+u\left(x_{m-k-1}, t\right)\right]\right\} \\
& +\mathcal{O}(h), \\
& { }_{\mathrm{RL}} D_{x, b}^{\alpha} u\left(x_{m}, t\right) \\
& =\frac{1}{\Gamma(3-\alpha) h^{\alpha}}\left\{\frac{(1-\alpha)(2-\alpha) u\left(x_{M}, t\right)}{(M-m)^{\alpha}}\right. \\
& +\frac{(2-\alpha)\left[u\left(x_{M}, t\right)-u\left(x_{M-1}, t\right)\right]}{(M-m)^{\alpha-1}} \\
& +\sum_{k=0}^{M-m-1} d_{k}^{(\alpha)}\left[u\left(x_{m+k-1}, t\right)\right. \\
& -2 u\left(x_{m+k}, t\right) \\
& \left.\left.+u\left(x_{m+k+1}, t\right)\right]\right\}
\end{aligned}
$$

$$
+\mathcal{O}(h)
$$

where $d_{k}^{(\alpha)}=(k+1)^{2-\alpha}-k^{2-\alpha}, k=0,1, \ldots, m-1$, or $k=$ $0,1, \ldots, M-m-1$.

Therefore, applying the above two formulas and (3) gives

$$
\begin{aligned}
\frac{\partial^{\alpha} u\left(x_{m}, t\right)}{\partial|x|^{\alpha}}=- & \frac{\Psi_{\alpha}}{\Gamma(3-\alpha) h^{\alpha}} \\
& \times\left\{\frac{(1-\alpha)(2-\alpha) u\left(x_{0}, t\right)}{m^{\alpha}}\right. \\
& +\frac{(2-\alpha)\left[u\left(x_{1}, t\right)-u\left(x_{0}, t\right)\right]}{m^{\alpha-1}} \\
& +\sum_{k=0}^{m-1} d_{k}^{(\alpha)}\left[u\left(x_{m-k+1}, t\right)-2 u\left(x_{m-k}, t\right)\right. \\
& +\frac{(1-\alpha)(2-\alpha) u\left(x_{M}, t\right)}{(M-m)^{\alpha}} \\
& +\frac{(2-\alpha)\left[u\left(x_{M}, t\right)-u\left(x_{M-1}, t\right)\right]}{(M-m)^{\alpha-1}} \\
& +\sum_{k=0}^{M-m-1} d_{k}^{(\alpha)}\left[u\left(x_{m+k-1}, t\right)-2 u\left(x_{m+k}, t\right)\right. \\
& \left.\left.\left.+x_{m+k+1}, t\right)\right]\right\}+\mathcal{O}(h),
\end{aligned}
$$

in which $d_{k}^{(\alpha)}$ is defined as above.

(IV) By the Spline Interpolation Method. In [26], Sousa proposed a second-order scheme by linear spline interpolation method for the left and right Riemann-Liouville derivatives,

$$
\begin{aligned}
{ }_{\mathrm{RL}} & D_{a, x}^{\alpha} u\left(x_{m}, t\right) \\
& =\frac{1}{\Gamma(4-\alpha) h^{\alpha}} \sum_{k=0}^{m+1} \bar{z}_{m, k}^{(\alpha)} u\left(x_{k}, t\right)+\mathcal{O}\left(h^{2}\right),
\end{aligned}
$$

where

$$
\bar{z}_{m, k}^{(\alpha)}= \begin{cases}\bar{c}_{m-1, k}-2 \bar{c}_{m, k}+\bar{c}_{m+1, k}, & k \leq m-1, \\ -2 \bar{c}_{m, k}+\bar{c}_{m+1, k}, & k=m, \\ \bar{c}_{m+1, k}, & k=m+1, \\ 0, & k>m+1,\end{cases}
$$

in which

$$
\begin{gathered}
\bar{c}_{j, k}= \begin{cases}(j-1)^{3-\alpha}-j^{2-\alpha}(j-3+\alpha), & k=0, \\
(j-k+1)^{3-\alpha}-2(j-k)^{3-\alpha} & \\
+(j-k-1)^{3-\alpha}, & 1 \leq k \leq j-1, \\
1, & k=j,\end{cases} \\
{ }_{\mathrm{RL}} D_{x, b}^{\alpha} u\left(x_{m}, t\right)=\frac{1}{\Gamma(4-\alpha) h^{\alpha}} \sum_{k=m-1}^{M} \widetilde{z}_{m, k}^{(\alpha)} u\left(x_{k}, t\right)+\mathcal{O}\left(h^{2}\right),
\end{gathered}
$$


where

$$
\widetilde{z}_{m, k}^{(\alpha)}= \begin{cases}0, & k<m-1, \\ \widetilde{c}_{m-1, m-1}, & k=m-1, \\ -2 \widetilde{c}_{m, m}+\widetilde{c}_{m-1, m}, & k=m, \\ \widetilde{c}_{m-1, k}-2 \widetilde{c}_{m, k}+\widetilde{c}_{m+1, k}, & m+1 \leq k \leq M,\end{cases}
$$

in which

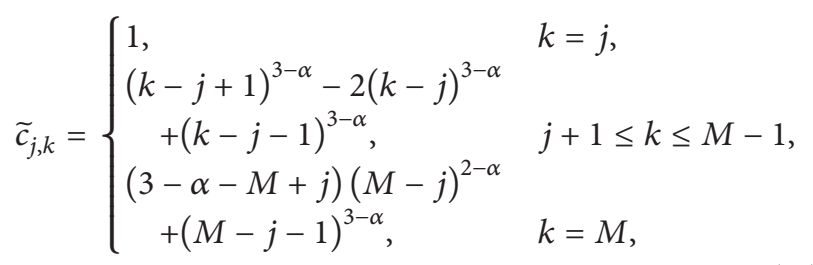

with $j=m-1, m, m+1$.

Combining (14), (16), and (3) gives

$$
\frac{\partial^{\alpha} u\left(x_{m}, t\right)}{\partial|x|^{\alpha}}=\frac{-\Psi_{\alpha}}{\Gamma(4-\alpha) h^{\alpha}} \sum_{k=0}^{M} z_{m, k}^{(\alpha)} u\left(x_{k}, t\right)+\mathcal{O}\left(h^{2}\right),
$$

where

$$
z_{m, k}^{(\alpha)}= \begin{cases}\bar{z}_{m, k}^{(\alpha)}, & k<m-1, \\ \bar{z}_{m, m-1}^{(\alpha)}+\widetilde{z}_{m, m-1}^{(\alpha)}, & k=m-1, \\ \bar{z}_{m, m}^{(\alpha)}+\widetilde{z}_{m, m}^{(\alpha)}, & k=m, \\ \bar{z}_{m, m+1}^{(\alpha)}+\widetilde{z}_{m, m+1}^{(\alpha)}, & k=m+1, \\ \widetilde{z}_{m, k}^{(\alpha)}, & k>m+1 .\end{cases}
$$

(V) By the Fractional Centered Difference Method. In [27], Ortigueira introduced a symmetrical fractional centered difference operator as follows:

$$
\begin{aligned}
\Delta_{h}^{\alpha} u(x, t)=\sum_{k=-\infty}^{\infty} & \frac{(-1)^{k} \Gamma(\alpha+1)}{\Gamma((\alpha / 2)-k+1) \Gamma((\alpha / 2)+k+1)} \\
& \times u(x-k h, t) .
\end{aligned}
$$

Later on, Çelik and Duman [23] proved that the above symmetrical fractional centered difference operator for the Riesz fractional derivative has the following relation:

$$
\frac{\partial^{\alpha} u\left(x_{m}, t\right)}{\partial|x|^{\alpha}}=-\frac{1}{h^{\alpha}} \Delta_{h}^{\alpha} u\left(x_{m}, t\right)+\mathcal{O}\left(h^{2}\right) .
$$

(VI) By the Weighted and Shifted Grünwald-Lentikov Formulas. In [28], Tian et al. proposed the second-order and third order numerical schemes for the left and right RiemannLiouville derivatives:

$$
\begin{aligned}
\mathrm{RL}_{a, x} D_{a, x}^{\alpha} u\left(x_{m}, t\right)= & \frac{\nu_{1}}{h^{\alpha}} \sum_{k=0}^{m+\ell_{1}} \Phi_{k}^{(\alpha)} u\left(x_{m-k+\ell_{1}}, t\right) \\
& +\frac{\nu_{2}}{h^{\alpha}} \sum_{k=0}^{m+\ell_{2}} \Phi_{k}^{(\alpha)} u\left(x_{m-k+\ell_{2}}, t\right)+\mathcal{O}\left(h^{2}\right), \\
\mathrm{RL}_{x, b} D^{\alpha} u\left(x_{m}, t\right)= & \frac{\nu_{1}}{h^{\alpha}} \sum_{k=0}^{M-m+\ell_{1}} \Phi_{k}^{(\alpha)} u\left(x_{m+k-\ell_{1}}, t\right) \\
& +\frac{\nu_{2}}{h^{\alpha}} \sum_{k=0}^{M-m+\ell_{2}} \Phi_{k}^{(\alpha)} u\left(x_{m+k-\ell_{2}}, t\right)+\mathcal{O}\left(h^{2}\right),
\end{aligned}
$$

where $\ell_{1}$ and $\ell_{2}$ are two arbitrary integers and $\ell_{1}-\ell_{2} \neq 0, v_{1}=$ $\left(\alpha-2 \ell_{2}\right) / 2\left(\ell_{1}-\ell_{2}\right)$, and $v_{2}=\left(2 \ell_{1}-\alpha\right) / 2\left(\ell_{1}-\ell_{2}\right)$.

And

$$
\begin{aligned}
\mathrm{RL}_{a, x} D_{a,}^{\alpha} u\left(x_{m}, t\right)= & \frac{\kappa_{1}}{h^{\alpha}} \sum_{k=0}^{m+\ell_{1}} \omega_{k}^{(\alpha)} u\left(x_{m-k+\ell_{1}}, t\right) \\
& +\frac{\kappa_{2}}{h^{\alpha}} \sum_{k=0}^{m+\ell_{2}} \omega_{k}^{(\alpha)} u\left(x_{m-k+\ell_{2}}, t\right) \\
& +\frac{\kappa_{3}}{h^{\alpha}} \sum_{k=0}^{m+\ell_{3}} \omega_{k}^{(\alpha)} u\left(x_{m-k+\ell_{3}}, t\right) \\
& +\mathcal{O}\left(h^{3}\right),
\end{aligned}
$$

$$
\begin{aligned}
\mathrm{RL} D_{x, b}^{\alpha} u\left(x_{m}, t\right)= & \frac{\kappa_{1}}{h^{\alpha}} \sum_{k=0}^{M-m+\ell_{1}} \omega_{k}^{(\alpha)} u\left(x_{m+k-\ell_{1}}, t\right) \\
& +\frac{\kappa_{2}}{h^{\alpha}} \sum_{k=0}^{M-m+\ell_{2}} \Phi_{k}^{(\alpha)} u\left(x_{m+k-\ell_{2}}, t\right) \\
& +\frac{\kappa_{3}}{h^{\alpha}} \sum_{k=0}^{M-m+\ell_{3}} \omega_{k}^{(\alpha)} u\left(x_{m+k-\ell_{3}}, t\right) \\
& +\mathcal{O}\left(h^{3}\right),
\end{aligned}
$$

in which $\ell_{1}, \ell_{2}$, and $\ell_{3}$ are three arbitrary integers and $\left(\ell_{1}-\right.$ $\left.\ell_{2}\right)\left(\ell_{2}-\ell_{3}\right)\left(\ell_{1}-\ell_{3}\right) \neq 0, \kappa_{1}=\left(12 \ell_{2} \ell_{3}-\left(6 \ell_{2}+6 \ell_{3}+1\right) \alpha+\right.$ $\left.3 \alpha^{2}\right) / 12\left(\ell_{2} \ell_{3}-\ell_{1} \ell_{2}-\ell_{1} \ell_{3}+\ell_{1}^{2}\right), \kappa_{2}=\left(12 \ell_{1} \ell_{3}-\left(6 \ell_{1}+6 \ell_{3}+\right.\right.$ $\left.1) \alpha+3 \alpha^{2}\right) / 12\left(\ell_{1} \ell_{3}-\ell_{1} \ell_{2}-\ell_{2} \ell_{3}+\ell_{2}^{2}\right)$, and $\kappa_{3}=\left(12 \ell_{1} \ell_{2}-\right.$ $\left.\left(6 \ell_{1}+6 \ell_{2}+1\right) \alpha+3 \alpha^{2}\right) / 12\left(\ell_{1} \ell_{2}-\ell_{1} \ell_{3}-\ell_{2} \ell_{3}+\ell_{3}^{2}\right)$. 
Naturally, we can obtain the following second-order and third order numerical formulas for the Riesz fractional derivative:

$$
\begin{aligned}
\frac{\partial^{\alpha} u\left(x_{m}, t\right)}{\partial|x|^{\alpha}}=-\frac{\Psi_{\alpha}}{h^{\alpha}}\left(v_{1} \sum_{k=0}^{m+\ell_{1}} \Phi_{k}^{(\alpha)} u\left(x_{m-k+\ell_{1}}, t\right)\right. & \\
& +v_{2} \sum_{k=0}^{m+\ell_{2}} \Phi_{k}^{(\alpha)} u\left(x_{m-k+\ell_{2}}, t\right) \\
& +v_{1} \sum_{k=0}^{M-m+\ell_{1}} \Phi_{k}^{(\alpha)} u\left(x_{m+k-\ell_{1}}, t\right) \\
& \left.+v_{2} \sum_{k=0}^{M-m+\ell_{2}} \Phi_{k}^{(\alpha)} u\left(x_{m+k-\ell_{2}}, t\right)\right) \\
+ &
\end{aligned}
$$

$$
\begin{aligned}
\frac{\partial^{\alpha} u\left(x_{m}, t\right)}{\partial|x|^{\alpha}}=-\frac{\Psi_{\alpha}}{h^{\alpha}}\left(\kappa_{1} \sum_{k=0}^{m+\ell_{1}} \omega_{k}^{(\alpha)} u\left(x_{m-k+\ell_{1}}, t\right)\right. & \\
& +\kappa_{2} \sum_{k=0}^{m+\ell_{2}} \Phi_{k}^{(\alpha)} u\left(x_{m-k+\ell_{2}}, t\right) \\
& +\kappa_{3} \sum_{k=0}^{m+\ell_{3}} \Phi_{k}^{(\alpha)} u\left(x_{m-k+\ell_{3}}, t\right) \\
& +\kappa_{1} \sum_{k=0}^{M-m+\ell_{1}} \omega_{k}^{(\alpha)} u\left(x_{m+k-\ell_{1}}, t\right) \\
& +\kappa_{2} \sum_{k=0}^{M-m+\ell_{2}} \omega_{k}^{(\alpha)} u\left(x_{m+k-\ell_{2}}, t\right) \\
& \left.+\kappa_{3} \sum_{k=0}^{M-m+\ell_{3}} \omega_{k}^{(\alpha)} u\left(x_{m+k-\ell_{3}}, t\right)\right) \\
+O\left(h^{3}\right), &
\end{aligned}
$$

respectively.

Here, we construct another second-order scheme and two fourth-order numerical schemes for the Riesz fractional derivative. In order to construct the new computational schemes, we introduce the following theorem.

Lemma 1 (see [29]). Let $\alpha>0, u(x, t) \in C_{0}^{\infty}(\mathbb{R})$ with respect to $x$. The Fourier transforms of the left and right RiemannLiouville fractional derivatives with respect to $x$ are

$$
\begin{aligned}
& \mathscr{F}_{x}\left({ }_{R L} D_{-\infty, x}^{\alpha} u(x, t)\right)=(i \omega)^{\alpha} \widehat{u}(\omega, t), \\
& \mathscr{F}_{x}\left({ }_{R L} D_{x, \infty}^{\alpha} u(x, t)\right)=(-i \omega)^{\alpha} \widehat{u}(\omega, t),
\end{aligned}
$$

where $\widehat{u}(\omega, t)$ denotes the Fourier transform of the function $u(x, t)$ with respect to $x$; that is,

$$
\widehat{u}(\omega, t)=\int_{\mathbb{R}} \exp (-i \omega x) u(x, t) d x .
$$

In [30], Tuan and Gorenflo introduced the following left fractional central difference operator:

$$
{ }_{C} \Delta_{-h}^{\alpha} u(x, t)=\sum_{k=0}^{\infty} \omega_{k}^{(\alpha)} u\left(x-\left(k-\frac{\alpha}{2}\right) h, t\right) .
$$

Similarly, we define the following right fractional central difference operator:

$$
{ }_{C} \Delta_{+h}^{\alpha} u(x, t)=\sum_{k=0}^{\infty} \omega_{k}^{(\alpha)} u\left(x+\left(k-\frac{\alpha}{2}\right) h, t\right) .
$$

Analogous to the integer-order finite difference formula, we define the following fractional average operator:

$$
\begin{aligned}
& \mu_{ \pm h}^{\alpha} u(x-s h, t) \\
& \quad=\frac{u(x \pm(s-(\alpha / 2)) h, t)+u(x \pm(s+(\alpha / 2)) h, t)}{2} .
\end{aligned}
$$

Then we can get the following fractional left and right average central difference operators based on (30), (31), and (32), respectively:

$$
\begin{aligned}
& { }_{\mathrm{AC}} \Delta_{-h}^{\alpha} u(x, t)=\mu_{-h}^{\alpha}\left({ }_{C} \Delta_{-h}^{\alpha} u(x, t)\right) \\
& =\sum_{j=0}^{\infty}(-1)^{j} \\
& \times\left(\begin{array}{c}
\alpha \\
j
\end{array}\right) \mu_{-h}^{\alpha}\left(u\left(x-\left(j-\frac{\alpha}{2}\right) h, t\right)\right) \\
& =\frac{1}{2} \sum_{j=0}^{\infty}(-1)^{j} \\
& \times\left(\begin{array}{c}
\alpha \\
j
\end{array}\right)(u(x-j h, t) \\
& +u(x-(j-\alpha) h, t)), \\
& { }_{\mathrm{AC}} \Delta_{+h}^{\alpha} u(x, t)=\mu_{+h}^{\alpha}\left({ }_{C} \Delta_{+h}^{\alpha} u(x, t)\right) \\
& =\sum_{j=0}^{\infty}(-1)^{j} \\
& \times\left(\begin{array}{c}
\alpha \\
j
\end{array}\right) \mu_{+h}^{\alpha}\left(u\left(x+\left(j-\frac{\alpha}{2}\right) h, t\right)\right) \\
& =\frac{1}{2} \sum_{j=0}^{\infty}(-1)^{j} \\
& \times\left(\begin{array}{c}
\alpha \\
j
\end{array}\right)(u(x+j h, t) \\
& +u(x-(j-\alpha) h, t)) .
\end{aligned}
$$


Here, we always assume that $\mu_{ \pm h}^{\alpha}$ can commute with the infinite summation.

For the fractional left and right average central difference operators defined in (33) and (34), one has the following result.

Theorem 2. Let $u(x, t)$ and the Fourier transform of ${ }_{R L} D_{-\infty, x}^{\alpha+2} u(x, t)$ and ${ }_{R L} D_{x,+\infty}^{\alpha+2} u(x, t)$ with respect to $x$ both be in $L_{1}(\mathbb{R})$; then

$$
\begin{aligned}
& { }_{R L} D_{-\infty, x}^{\alpha} u(x, t)=\frac{A_{C} \Delta_{-h}^{\alpha} u(x, t)}{h^{\alpha}}+\mathcal{O}\left(h^{2}\right), \\
& { }_{R L} D_{x,+\infty}^{\alpha} u(x, t)=\frac{A_{C} \Delta_{+h}^{\alpha} u(x, t)}{h^{\alpha}}+\mathcal{O}\left(h^{2}\right)
\end{aligned}
$$

uniformly holds for $x \in \mathbb{R}$.

Proof. Here, we only prove (35). As $u(x, t)$ with respect to $x$ belongs to $L_{1}(\mathbb{R})$, then the Fourier transform of the fractional average central difference operator (33) exists and has the following form:

$$
\begin{aligned}
& \mathscr{F}_{x}\left\{\frac{\mathrm{AC} \Delta_{-h^{\alpha}}^{\alpha} u(x, t)}{h^{\alpha}} ; \omega\right\} \\
& =\frac{1}{2 h^{\alpha}} \sum_{j=0}^{\infty}(-1)^{j} \\
& \quad \times\left(\begin{array}{c}
\alpha \\
j
\end{array}\right)(\exp (-i \omega j h) \\
& \quad+\exp (-i \omega(j-\alpha) h)) \widehat{u}(\omega, t) \\
& =\frac{1}{h^{\alpha}}\left(\sum_{j=0}^{\infty}(-1)^{j}\left(\begin{array}{c}
\alpha \\
j
\end{array}\right) \exp (-i j \omega h)\right) \\
& \quad \times\left(\frac{1+\exp (i \omega \alpha h)}{2}\right) \widehat{u}(\omega, t) \\
& =(i \omega)^{\alpha}\left(\frac{1-\exp (-i \omega h)}{i \omega h}\right)^{\alpha}\left(\frac{1+\exp (i \omega \alpha h)}{2}\right) \widehat{u}(\omega, t) .
\end{aligned}
$$

Note that the function $((1-\exp (-i \omega h)) / i \omega h)^{\alpha}((1+$ $\exp (i \omega \alpha h)) / 2$ ) has the following Taylor expansion:

$$
\begin{gathered}
\left(\frac{1-\exp (-i \omega h)}{i \omega h}\right)^{\alpha}\left(\frac{1+\exp (i \omega \alpha h)}{2}\right) \\
=1+\frac{\alpha(3 \alpha+1)}{24}(i \omega h)^{2}+\mathcal{O}(|i \omega h|)^{4}
\end{gathered}
$$

If we denote

$$
\begin{aligned}
\widehat{\phi}(\omega, h)= & \mathscr{F}_{x}\left\{\frac{\mathrm{AC}_{-h}^{\alpha} u(x, t)}{h^{\alpha}} ; \omega\right\} \\
& -\mathscr{F}_{x}\left({ }_{\mathrm{RL}} D_{-\infty, x}^{\alpha} u(x, t)\right),
\end{aligned}
$$

then from (36), (37), and Lemma 1, we have

$$
|\widehat{\phi}(\omega, h)| \leq C_{1} h^{2}\left|(i \omega)^{\alpha+2} \widehat{u}(\omega, t)\right|
$$
is,

In light of the condition $\mathscr{F}\left({ }_{\mathrm{RL}} D_{-\infty, x}^{\alpha+2} u(x, t)\right) \in L_{1}(\mathbb{R})$, that

$$
\int_{\mathbb{R}}\left|\mathscr{F}\left({ }_{\mathrm{RL}} D_{-\infty, x}^{\alpha+2} u(x, t)\right)\right| d \omega<C_{2}
$$

we obtain

$$
\begin{aligned}
& \left|\frac{\mathrm{AC}_{-h}^{\alpha} u(x, t)}{h^{\alpha}}-{ }_{\mathrm{RL}} D_{-\infty, x}^{\alpha} u(x, t)\right| \\
& \quad=|\phi(\omega, h)| \\
& \quad=\frac{1}{2 \pi}\left|\int_{\mathbb{R}} \exp (i \omega h) \widehat{\phi}(\omega, h) d \omega\right| \\
& \leq \frac{1}{2 \pi} \int_{\mathbb{R}}|\widehat{\phi}(\omega, h)| d \omega \\
& \leq \frac{C_{1}}{2 \pi}\left(\int_{\mathbb{R}}\left|(i \omega)^{\alpha+2} \widehat{u}(\omega, t)\right| d \omega\right) h^{2} \\
& \quad=\frac{C_{1}}{2 \pi}\left(\int_{\mathbb{R}}\left|\mathscr{F}\left({ }_{\mathrm{RL}} D_{-\infty, x}^{\alpha+2} u(x, t)\right)\right| d \omega\right) h^{2} \\
& \quad \leq C h^{2} \\
& \quad=\mathscr{O}\left(h^{2}\right),
\end{aligned}
$$

where $C=C_{1} C_{2} / 2 \pi$. This finishes the proof.

Next, we construct fourth-order difference scheme for the left and right Riemann-Liouville derivatives based on (33) and (34) by the following theorem.

Theorem 3. Let $u(x, t)$ and the Fourier transform of ${ }_{R L} D_{-\infty, x}^{\alpha+4} u(x, t)$ and ${ }_{R L} D_{x,+\infty}^{\alpha+4} u(x, t)$ with respect to $x$ both be in $L_{1}(\mathbb{R})$; then

$$
\begin{aligned}
& { }_{R L} D_{-\infty, x}^{\alpha} u(x, t)=\frac{1}{h^{\alpha}} \frac{A C^{\Delta_{-h}^{\alpha}} u(x, t)}{1+(\alpha(3 \alpha+1) / 24) \delta_{x}^{2}}+\mathcal{O}\left(h^{4}\right), \\
& { }_{R L} D_{x,+\infty}^{\alpha} u(x, t)=\frac{1}{h^{\alpha}} \frac{A_{C} \Delta_{+h}^{\alpha} u(x, t)}{1+(\alpha(3 \alpha+1) / 24) \delta_{x}^{2}}+\mathcal{O}\left(h^{4}\right)
\end{aligned}
$$

uniformly holds for $x \in \mathbb{R}$, where $\delta_{x}^{2}$ denotes second-order central difference operator with respect to $x$ and is defined by $\delta_{x}^{2} u\left(x_{j}, t\right)=u\left(x_{j+1}, t\right)-2 u\left(x_{j}, t\right)+u\left(x_{j-1}, t\right)$.

Proof. It is similar to Theorem 2. 
Combining (3) and Theorems 2 and 3, we can get the following high-order difference schemes for the Riesz derivative:

$$
\begin{gathered}
\frac{\partial^{\alpha} u(x, t)}{\partial|x|^{\alpha}}=-\frac{\Psi_{\alpha}}{2 h^{\alpha}}\left[\sum_{j=0}^{\infty}(-1)^{j}\left(\begin{array}{c}
\alpha \\
j
\end{array}\right)\right. \\
\times(u(x-j h, t) \\
+u(x-(j-\alpha) h, t)) \\
+\sum_{j=0}^{\infty}(-1)^{j}\left(\begin{array}{c}
\alpha \\
j
\end{array}\right) \\
\times(u(x+j h, t) \\
+\mathcal{O}\left(h^{2}\right),
\end{gathered}
$$$$
\frac{\partial^{\alpha} u(x, t)}{\partial|x|^{\alpha}}=-\frac{\Psi_{\alpha}}{2 h^{\alpha}}\left[\sum_{j=0}^{\infty}(-1)^{j}\left(\begin{array}{c}
\alpha \\
j
\end{array}\right)\right.
$$$$
\times \frac{u(x-j h, t)+u(x-(j-\alpha) h, t)}{1+(\alpha(3 \alpha+1) / 24) \delta_{x}^{2}}
$$$$
+\sum_{j=0}^{\infty}(-1)^{j}\left(\begin{array}{c}
\alpha \\
j
\end{array}\right)
$$

$$
\begin{aligned}
& \left.\times \frac{u(x+j h, t)+u(x+(j-\alpha) h, t)}{1+(\alpha(3 \alpha+1) / 24) \delta_{x}^{2}}\right] \\
& +\mathcal{O}\left(h^{4}\right) .
\end{aligned}
$$

Moreover, let

$$
\widetilde{u}(x, t)= \begin{cases}u(x, t), & x \in[a, b], \\ 0, & x \notin[a, b] ;\end{cases}
$$

then formulas (43) and (44) change into

$$
\begin{aligned}
& \frac{\partial^{\alpha} u(x, t)}{\partial|x|^{\alpha}} \\
&=-\frac{\Psi_{\alpha}}{2 h^{\alpha}}\left[\sum_{j=0}^{[(x-\alpha) / h]}(-1)^{j}\left(\begin{array}{c}
\alpha \\
j
\end{array}\right)\right. \\
& \times(u(x-j h, t) \\
&+u(x-(j-\alpha) h, t))
\end{aligned}
$$$$
+\sum_{j=0}^{[(b-x) / h]}(-1)^{j}\left(\begin{array}{l}
\alpha \\
j
\end{array}\right)
$$$$
\times(u(x+j h, t)
$$$$
+u(x+(j-\alpha) h, t))]
$$$$
+\mathcal{O}\left(h^{2}\right)
$$

$$
\begin{aligned}
& \frac{\partial^{\alpha} u(x, t)}{\partial|x|^{\alpha}} \\
& =-\frac{\Psi_{\alpha}}{2 h^{\alpha}}\left[\sum_{j=0}^{[(x-\alpha) / h]}(-1)^{j}\left(\begin{array}{c}
\alpha \\
j
\end{array}\right)\right. \\
& \quad \times \frac{u(x-j h, t)+u(x-(j-\alpha) h, t)}{1+(\alpha(3 \alpha+1) / 24) \delta_{x}^{2}} \\
& +\sum_{j=0}^{[(b-x) / h]}(-1)^{j}\left(\begin{array}{c}
\alpha \\
j
\end{array}\right) \\
& \left.\times \frac{u(x+j h, t)+u(x+(j-\alpha) h, t)}{1+(\alpha(3 \alpha+1) / 24) \delta_{x}^{2}}\right] \\
& +\mathcal{O}\left(h^{4}\right) .
\end{aligned}
$$

Finally, we derive another fourth-order numerical method for the Riesz fractional derivative which is presented in the following theorem.

Theorem 4. Let $u(x, t)$ lie in $C^{7}(\mathbb{R})$ whose partial derivatives up to order seven with respect to $x$ belong to $L_{1}(\mathbb{R})$. Set

$$
\mathscr{L}_{\theta} u(x, t)=\sum_{k=-\infty}^{\infty} g_{k}^{(\alpha)} u(x-(k+\theta) h, t), \quad \theta=-1,0,1,
$$

in which

$$
g_{k}^{(\alpha)}=\frac{(-1)^{k} \Gamma(\alpha+1)}{\Gamma((\alpha / 2)-k+1) \Gamma((\alpha / 2)+k+1)} ;
$$

then one has

$$
\begin{aligned}
& \frac{\partial^{\alpha} u(x, t)}{\partial|x|^{\alpha}} \\
& =\frac{1}{h^{\alpha}}\left[\frac{\alpha}{24} \mathscr{L}_{-1} u(x, t)-\left(1+\frac{\alpha}{12}\right) \mathscr{L}_{0} u(x, t)\right. \\
& \left.\quad+\frac{\alpha}{24} \mathscr{L}_{1} u(x, t)\right]+O\left(h^{4}\right) .
\end{aligned}
$$


Proof. Here, we use the Fourier transform method to prove it [23]. From [27], we know that the generating function with coefficients $g_{k}^{(\alpha)}$ satisfies

$$
\left|2 \sin \left(\frac{x}{2}\right)\right|^{\alpha}=\sum_{k=-\infty}^{\infty} g_{k}^{(\alpha)} \exp (i k x)
$$

From (3) and Lemma 1, we get the Fourier transform of the Riesz fractional derivative as follows:

$$
\begin{aligned}
\mathscr{F}_{x}\left\{\frac{\partial^{\alpha} u(x, t)}{\partial|x|^{\alpha}} ; \omega\right\} & =-\Psi_{\alpha}\left[(i \omega)^{\alpha}+(-i \omega)^{\alpha}\right] \widehat{u}(\omega, t) \\
& =-|\omega|^{\alpha} \widehat{u}(\omega, t) .
\end{aligned}
$$

Applying the Fourier transform to the difference operator

$$
\frac{1}{h^{\alpha}}\left[\frac{\alpha}{24} \mathscr{L}_{-1} u(x, t)-\left(1+\frac{\alpha}{12}\right) \mathscr{L}_{0} u(x, t)+\frac{\alpha}{24} \mathscr{L}_{1} u(x, t)\right]
$$

with respect to $x$ and using (51) give

$$
\begin{gathered}
\mathscr{F}_{x}\left\{\frac { 1 } { h ^ { \alpha } } \left[\frac{\alpha}{24} \mathscr{L}_{-1} u(x, t)-\left(1+\frac{\alpha}{12}\right) \mathscr{L}_{0} u(x, t)\right.\right. \\
\left.\left.\quad+\frac{\alpha}{24} \mathscr{L}_{1} u(x, t)\right] ; \omega\right\} \\
=\frac{1}{h^{\alpha}}\left[\frac{\alpha}{24} \sum_{k=-\infty}^{\infty} g_{k}^{(\alpha)} \exp (-i(k-1) \omega h) \widehat{u}(\omega, t)\right. \\
-\left(1+\frac{\alpha}{12}\right) \sum_{k=-\infty}^{\infty} g_{k}^{(\alpha)} \exp (-i k \omega h) \widehat{u}(\omega, t) \\
\left.+\frac{\alpha}{24} \sum_{k=-\infty}^{\infty} g_{k}^{(\alpha)} \exp (-i(k+1) \omega h) \widehat{u}(\omega, t)\right] \\
=-\frac{1}{h^{\alpha}}\left[1+\frac{\alpha}{12}(1-\cos (\omega h))\right]\left|2 \sin \left(\frac{\omega h}{2}\right)\right|^{\alpha} \widehat{u}(\omega, t) .
\end{gathered}
$$

Set

$$
\begin{aligned}
|\omega|^{\alpha} \widehat{u}(\omega, t) & \\
=\widehat{\mathscr{C}}(h, \omega)-\mathscr{F}_{x}\left\{\frac{1}{h^{\alpha}}[\right. & \frac{\alpha}{24} \mathscr{L}_{-1} u(x, t) \\
& -\left(1+\frac{\alpha}{12}\right) \mathscr{L}_{0} u(x, t) \\
& \left.\left.+\frac{\alpha}{24} \mathscr{L}_{1} u(x, t)\right] ; \omega\right\} ;
\end{aligned}
$$

then

$$
\begin{aligned}
& \widehat{\mathscr{C}}(h, \omega)=|\omega|^{\alpha}\{1-\left[1+\frac{\alpha}{12}(1-\cos (\omega h))\right] \\
& \times\left.\left|\frac{2 \sin (\omega h / 2)}{\omega h}\right|^{\alpha}\right\} \widehat{u}(\omega, t) \\
&=|\omega|^{\alpha}\left\{1-\left[1+\frac{\alpha}{24}(\omega h)^{2}-\frac{\alpha}{288}(\omega h)^{4}+O(\omega h)^{6}\right]\right. \\
& \cdot\left[1-\frac{\alpha}{24}(\omega h)^{2}+\alpha\left(\frac{1}{1920}+\frac{\alpha-1}{1152}\right)(\omega h)^{4}\right. \\
&\left.\left.+O(\omega h)^{6}\right]\right\} \widehat{u}(\omega, t) \\
&=-|\omega|^{\alpha}\left\{\alpha\left(\frac{\alpha}{1152}+\frac{11}{2880}\right)(\omega h)^{4}\right. \\
&\left.-O(\omega h)^{6}\right\} \widehat{u}(\omega, t) .
\end{aligned}
$$

Since $u(x, t) \in C^{7}(\mathbb{R})$ and its partial derivatives up to order seven with respect to $x$ belong to $L_{1}(\mathbb{R})$, there exists a positive constant $\widetilde{C}_{1}$ such that

$$
|\widehat{u}(\omega, t)| \leq \widetilde{C}_{1}(1+|\omega|)^{-7} .
$$

So, using (56) and (57) leads to

$$
\begin{aligned}
|\widehat{\mathscr{C}}(h, \omega)| & \leq \widetilde{C}_{2} h^{4}|\omega|^{4+\alpha}|\widehat{u}(\omega, t)| \\
& \leq \widetilde{C}_{2} h^{4}(1+|\omega|)^{4+\alpha}|\widehat{u}(\omega, t)| \\
& \leq \widetilde{C}_{3} h^{4}(1+|\omega|)^{\alpha-3}
\end{aligned}
$$

where $\widetilde{C}_{3}=\widetilde{C}_{1} \widetilde{C}_{2}$.

At this moment, taking the inverse Fourier transformation in both sides of (55) and noting (52) give

$$
\begin{gathered}
\frac{\partial^{\alpha} u(x, t)}{\partial|x|^{\alpha}}=\frac{1}{h^{\alpha}}\left[\frac{\alpha}{24} \mathscr{L}_{-1} u(x, t)-\left(1+\frac{\alpha}{12}\right) \mathscr{L}_{0} u(x, t)\right. \\
\left.+\frac{\alpha}{24} \mathscr{L}_{1} u(x, t)\right]-\mathscr{C}(h, \omega) .
\end{gathered}
$$

In view of (58), we have

$$
\begin{aligned}
|\mathscr{C}(h, x)| & =\frac{1}{2 \pi}\left|\int_{\mathbb{R}} \widehat{\mathscr{C}}(h, \omega) \exp (i \omega x) d \omega\right| \\
& \leq \frac{1}{2 \pi} \int_{\mathbb{R}}|\widehat{\mathscr{C}}(h, \omega)| d \omega \\
& \leq \frac{\widetilde{C_{3}}}{2 \pi}\left(\int_{\mathbb{R}}(1+|\omega|)^{\alpha-3} d \omega\right) h^{4} \\
& =\widetilde{C} h^{4}
\end{aligned}
$$


where $\widetilde{C}=\widetilde{C}_{3} /(2-\alpha) \pi$; that is to say,

$$
\begin{gathered}
\frac{\partial^{\alpha} u(x, t)}{\partial|x|^{\alpha}}=\frac{1}{h^{\alpha}}\left[\frac{\alpha}{24} \mathscr{L}_{-1} u(x, t)-\left(1+\frac{\alpha}{12}\right) \mathscr{L}_{0} u(x, t)\right. \\
\left.+\frac{\alpha}{24} \mathscr{L}_{1} u(x, t)\right]+O\left(h^{4}\right) .
\end{gathered}
$$

This finishes the proof.

Furthermore, (61) can be rewritten as

$$
\begin{aligned}
\frac{\partial^{\alpha} u(x, t)}{\partial|x|^{\alpha}}= & \frac{\alpha}{24 h^{\alpha}} \sum_{k=-\infty}^{\infty} g_{k}^{(\alpha)} u(x-(k+1) h, t) \\
& -\left(1+\frac{\alpha}{12}\right) \frac{1}{h^{\alpha}} \sum_{k=-\infty}^{\infty} g_{k}^{(\alpha)} u(x-k h, t) \\
& +\frac{\alpha}{24 h^{\alpha}} \sum_{k=-\infty}^{\infty} g_{k}^{(\alpha)} u(x-(k-1) h, t)+O\left(h^{4}\right) .
\end{aligned}
$$

Combining (45), one can get

$$
\begin{aligned}
\frac{\partial^{\alpha} u\left(x_{m}, t\right)}{\partial|x|^{\alpha}}= & \frac{\alpha}{24 h^{\alpha}} \sum_{k=-M+m+1}^{m-1} g_{k}^{(\alpha)} u\left(x_{m-(k+1)}, t\right) \\
& +\frac{\alpha}{24 h^{\alpha}} \sum_{k=-M+m+1}^{m-1} g_{k}^{(\alpha)} u\left(x_{m-(k-1)}, t\right) \\
& -\left(1+\frac{\alpha}{12}\right) \frac{1}{h^{\alpha}} \sum_{k=-M+m+1}^{m-1} g_{k}^{(\alpha)} u\left(x_{m-k}, t\right) \\
& +\mathcal{O}\left(h^{4}\right) .
\end{aligned}
$$

\section{The Numerical Method for the Space Riesz Fractional Diffusion Equation}

In this section, we only develop the fourth-order numerical method for the Riesz fractional diffusion equation (1). The second-order methods derived in the preceding section for (1) are easy so they are omitted here.

By Taylor expansion, one has

$$
\begin{aligned}
u\left(x_{m}, t_{n-1}\right)= & u\left(x_{m}, t_{n}\right)-\tau \frac{\partial u\left(x_{m}, t_{n}\right)}{\partial t} \\
& +\frac{\tau^{2}}{2} \frac{\partial^{2} u\left(x_{m}, t_{n}\right)}{\partial t^{2}}+\mathcal{O}\left(\tau^{3}\right), \\
\frac{\partial u\left(x_{m}, t_{n-1}\right)}{\partial t}= & \frac{\partial u\left(x_{m}, t_{n}\right)}{\partial t} \\
& -\tau \frac{\partial^{2} u\left(x_{m}, t_{n}\right)}{\partial t^{2}}+\mathcal{O}\left(\tau^{2}\right) .
\end{aligned}
$$

Furthermore, from (65), one gets

$$
\frac{\partial^{2} u\left(x_{m}, t_{n}\right)}{\partial t^{2}}=\frac{1}{\tau}\left(\frac{\partial u\left(x_{m}, t_{n}\right)}{\partial t}-\frac{\partial u\left(x_{m}, t_{n-1}\right)}{\partial t}\right)+\mathcal{O}(\tau)
$$

Substituting (66) into (64) yields

$$
\begin{aligned}
& \frac{\tau}{2}\left(\frac{\partial u\left(x_{m}, t_{n}\right)}{\partial t}+\frac{\partial u\left(x_{m}, t_{n-1}\right)}{\partial t}\right) \\
& \quad=u\left(x_{m}, t_{n}\right)-u\left(x_{m}, t_{n-1}\right)+\mathcal{O}\left(\tau^{3}\right) .
\end{aligned}
$$

From (1) and (67), we have

$$
\begin{aligned}
2 u\left(x_{m}, t_{n}\right)-\tau K \frac{\partial^{\alpha} u\left(x_{m}, t_{n}\right)}{\partial|x|^{\alpha}} \\
=2 u\left(x_{m}, t_{n-1}\right)+\tau K \frac{\partial^{\alpha} u\left(x_{m}, t_{n-1}\right)}{\partial|x|^{\alpha}}+\tau f\left(x_{m}, t_{n}\right) \\
\quad+\tau f\left(x_{m}, t_{n-1}\right)+\mathcal{O}\left(\tau^{3}\right) .
\end{aligned}
$$

Let $u_{m}^{n}$ be the approximation solution of $u\left(x_{m}, t_{n}\right)$. Substituting (63) into (68) and removing the high-order terms, one has the following finite difference scheme for (1):

$$
\begin{aligned}
u_{m}^{n}- & \mu_{1} \sum_{k=-M+m+1}^{m-1} g_{k}^{(\alpha)} u_{m-k-1}^{n} \\
& -\mu_{1} \sum_{k=-M+m+1}^{m-1} g_{k}^{(\alpha)} u_{m-k+1}^{n} \\
& +\mu_{2} \sum_{k=-M+m+1}^{m-1} g_{k}^{(\alpha)} u_{m-k}^{n} \\
= & u_{m}^{n-1}+\mu_{1} \sum_{k=-M+m+1}^{m-1} g_{k}^{(\alpha)} u_{m-k-1}^{n-1} \\
& +\mu_{1} \sum_{k=-M+m+1}^{m-1} g_{k}^{(\alpha)} u_{m-k+1}^{n-1} \\
& -\mu_{2} \sum_{k=-M+m+1}^{m-1} g_{k}^{(\alpha)} u_{m-k}^{n-1}+\frac{\tau}{2} f_{m}^{n}+\frac{\tau}{2} f_{m}^{n-1}
\end{aligned}
$$

where $\mu_{1}=\alpha \tau K / 48 h^{\alpha}$ and $\mu_{2}=\left(\tau K / 2 h^{\alpha}\right)(1+(\alpha / 12))$.

Denoting

$$
U^{n}=\left(u_{1}^{n}, u_{2}^{n}, \ldots, u_{M-1}^{n}\right)^{T}, \quad F^{n}=\left(f_{1}^{n}, f_{2}^{n}, \ldots, f_{M-1}^{n}\right)^{T}
$$

then system (69) can be written in the following matrix form:

$$
(I+H) U^{n}=(I-H) U^{n-1}+\frac{\tau}{2} F^{n}+\frac{\tau}{2} F^{n-1},
$$


where $I$ is a $(M-1) \times(M-1)$ identity matrix, $H=\mu_{2} G-$ $\mu_{1} G^{+}-\mu_{1} G^{-}$,

$$
G=\left(\begin{array}{ccccccc}
g_{0}^{(\alpha)} & g_{-1}^{(\alpha)} & g_{-2}^{(\alpha)} & \cdots & g_{4-M}^{(\alpha)} & g_{3-M}^{(\alpha)} & g_{-M}^{(\alpha)} \\
g_{1}^{(\alpha)} & g_{0}^{(\alpha)} & g_{-1}^{(\alpha)} & g_{-2}^{(\alpha)} & \cdots & g_{4-M}^{(\alpha)} & g_{3-M}^{(\alpha)} \\
g_{2}^{(\alpha)} & g_{1}^{(\alpha)} & g_{0}^{(\alpha)} & g_{-1}^{(\alpha)} & g_{-2}^{(\alpha)} & \cdots & g_{-M}^{(\alpha)} \\
\vdots & \ddots & \ddots & \ddots & \ddots & \ddots & \vdots \\
g_{M-4}^{(\alpha)} & \cdots & g_{2}^{(\alpha)} & g_{1}^{(\alpha)} & g_{0}^{(\alpha)} & g_{-1}^{(\alpha)} & g_{-2}^{(\alpha)} \\
g_{M-3}^{(\alpha)} & g_{M-4}^{(\alpha)} & \cdots & g_{2}^{(\alpha)} & g_{1}^{(\alpha)} & g_{0}^{(\alpha)} & g_{-1}^{(\alpha)} \\
g_{M-2}^{(\alpha)} & g_{M-3}^{(\alpha)} & g_{M-4}^{(\alpha)} & \cdots & g_{2}^{(\alpha)} & g_{1}^{(\alpha)} & g_{0}^{(\alpha)}
\end{array}\right),
$$

$$
G^{+}=\left(\begin{array}{ccccccc}
0 & g_{0}^{(\alpha)} & g_{-1}^{(\alpha)} & \cdots & g_{5-M}^{(\alpha)} & g_{4-M}^{(\alpha)} & g_{3-M}^{(\alpha)} \\
0 & g_{1}^{(\alpha)} & g_{0}^{(\alpha)} & g_{-1}^{(\alpha)} & \cdots & g_{5-M}^{(\alpha)} & g_{4-M}^{(\alpha)} \\
0 & g_{2}^{(\alpha)} & g_{1}^{(\alpha)} & g_{0}^{(\alpha)} & g_{-1}^{(\alpha)} & \cdots & g_{5-M}^{(\alpha)} \\
\vdots & \ddots & \ddots & \ddots & \ddots & \ddots & \vdots \\
0 & \cdots & g_{3}^{(\alpha)} & g_{2}^{(\alpha)} & g_{1}^{(\alpha)} & g_{0}^{(\alpha)} & g_{-1}^{(\alpha)} \\
0 & g_{M-3}^{(\alpha)} & \cdots & g_{3}^{(\alpha)} & g_{2}^{(\alpha)} & g_{1}^{(\alpha)} & g_{0}^{(\alpha)} \\
0 & g_{M-2}^{(\alpha)} & g_{M-3}^{(\alpha)} & \cdots & g_{3}^{(\alpha)} & g_{2}^{(\alpha)} & g_{1}^{(\alpha)}
\end{array}\right)
$$

$$
G^{-}=\left(\begin{array}{ccccccc}
g_{-1}^{(\alpha)} & g_{-2}^{(\alpha)} & g_{-3}^{(\alpha)} & \cdots & g_{3-M}^{(\alpha)} & g_{2-M}^{(\alpha)} & 0 \\
g_{0}^{(\alpha)} & g_{-1}^{(\alpha)} & g_{-2}^{(\alpha)} & g_{-3}^{(\alpha)} & \cdots & g_{3-M}^{(\alpha)} & 0 \\
g_{1}^{(\alpha)} & g_{0}^{(\alpha)} & g_{-1}^{(\alpha)} & g_{-2}^{(\alpha)} & g_{-3}^{(\alpha)} & \cdots & 0 \\
\vdots & \ddots & \ddots & \ddots & \ddots & \ddots & \vdots \\
g_{M-5}^{(\alpha)} & \cdots & g_{1}^{(\alpha)} & g_{0}^{(\alpha)} & g_{-1}^{(\alpha)} & g_{-2}^{(\alpha)} & 0 \\
g_{M-4}^{(\alpha)} & g_{M-5}^{(\alpha)} & \cdots & g_{1}^{(\alpha)} & g_{0}^{(\alpha)} & g_{-1}^{(\alpha)} & 0 \\
g_{M-3}^{(\alpha)} & g_{M-4}^{(\alpha)} & g_{M-5}^{(\alpha)} & \cdots & g_{1}^{(\alpha)} & g_{0}^{(\alpha)} & 0
\end{array}\right)
$$

$$
\begin{aligned}
g_{k}^{(\alpha)} & =\frac{(-1)^{k} \Gamma(\alpha+1)}{\Gamma((\alpha / 2)-k+1) \Gamma((\alpha / 2)+k+1)}, \\
& k=2-M, 3-M, \ldots, M-3, M-2 .
\end{aligned}
$$

\section{Stability Analysis}

Now we perform the detailed stability analysis for the difference scheme (71). Firstly, we introduce some lemmas and a definition for the following discussion.
Lemma 5 (see [31]). Let $\mathscr{A}$ be an $M-1$ order positive definite matrix. Then for any parameter $\sigma \geq 0$, the following two inequalities:

$$
\begin{gathered}
\left\|(I+\sigma \mathscr{A})^{-1}\right\|_{\infty} \leq 1, \\
\left\|(I+\sigma \mathscr{A})^{-1}(I-\sigma \mathscr{A})\right\|_{\infty} \leq 1,
\end{gathered}
$$

hold.

Definition 6 (see [32]). Let Toeplitz matrix $\mathbb{Q}_{M}$ have the following form:

$$
Q_{M}=\left(\begin{array}{ccccc}
\varrho_{0} & \varrho_{-1} & \cdots & \varrho_{2-M} & \varrho_{1-M} \\
\varrho_{1} & \varrho_{0} & \varrho_{-1} & \cdots & \varrho_{2-M} \\
\vdots & \varrho_{1} & \varrho_{0} & \ddots & \vdots \\
\varrho_{M-2} & \cdots & \ddots & \ddots & \varrho_{-1} \\
\varrho_{M-1} & \varrho_{M-2} & \cdots & \varrho_{1} & \varrho_{0}
\end{array}\right) .
$$

If the diagonals $\left\{\varrho_{k}\right\}_{k=-M+1}^{M-1}$ are the Fourier coefficients of function $U(x, t)$, that is,

$$
\varrho_{k}=\frac{1}{2 \pi} \int_{-\pi}^{\pi} U(x, t) e^{-i k x} d x,
$$

then the function $U(x, t)$ is called the generating function of $Q_{M}$.

Lemma 7 (see [33]). For the above Toeplitz matrix $\mathbb{Q}_{M}$, let $U(x, t)$ be a $2 \pi$-periodic continuous real-valued function defined on $[-\pi, \pi]$. Denote $\lambda_{\min }\left(Q_{M}\right)$ and $\lambda_{\max }\left(Q_{M}\right)$ as the smallest and largest eigenvalues of $\mathbb{Q}_{M}$, respectively. Then one has

$$
F_{\min } \leq \lambda_{\min }\left(\mathscr{Q}_{M}\right) \leq \lambda_{\max }\left(\mathscr{Q}_{M}\right) \leq F_{\max },
$$

where $F_{\min }$ and $F_{\max }$ are the minimum and maximum values of $U(x, t)$. Moreover, if $F_{\min }<F_{\max }$, then all eigenvalues of $Q_{M}$ satisfy

$$
F_{\text {min }}<\lambda\left(Q_{M}\right)<F_{\max },
$$

for all $M>0$. And furthermore if $F_{\min } \geq 0$, then $\mathbb{Q}_{M}$ is positive definite.

Theorem 8. The matrix $H$ is positive definite.

Proof. From Definition 6, we know that the generating functions of the matrices $G, G^{+}$, and $G^{-}$are

$$
\begin{aligned}
& F_{G}(x)=\sum_{k=-\infty}^{\infty} g_{k}^{(\alpha)} e^{i k x}, \\
& F_{G^{+}}(x)=\sum_{k=-\infty}^{\infty} g_{k}^{(\alpha)} e^{i(k+1) x}, \\
& F_{G^{-}}(x)=\sum_{k=-\infty}^{\infty} g_{k}^{(\alpha)} e^{i(k-1) x},
\end{aligned}
$$

respectively. 
Therefore the generating function of the matrix $H$ is

$$
F_{H}(x)=\mu_{2} F_{G}(x)-\mu_{1} F_{G^{+}}(x)-\mu_{1} F_{G^{-}}(x)
$$

that is,

$$
\begin{aligned}
F_{H}(x)= & \mu_{2} \sum_{k=-\infty}^{\infty} g_{k}^{(\alpha)} e^{i k x}-\mu_{1} \sum_{k=-\infty}^{\infty} g_{k}^{(\alpha)} e^{i(k+1) x} \\
& -\mu_{1} \sum_{k=-\infty}^{\infty} g_{k}^{(\alpha)} e^{i(k-1) x} \\
= & \left(\mu_{2}-2 \mu_{1} \cos x\right) \sum_{k=-\infty}^{\infty} g_{k}^{(\alpha)} e^{i k x} \\
= & \frac{K \tau}{12 h^{\alpha}}\left[6+\alpha \sin ^{2}\left(\frac{x}{2}\right)\right]\left|2 \sin \left(\frac{x}{2}\right)\right|^{\alpha} \geq 0 .
\end{aligned}
$$

It immediately follows that $H$ is a positive definite matrix in view of Lemma 7.

Theorem 9. The difference scheme (71) is unconditionally stable.

Proof. Let $U^{n}$ and $\widetilde{U}^{n}$ be the exact and numerical solutions of difference equation (71). Since the matrix $(I+H)^{-1}$ is invertible, then we can obtain the following error equation:

$$
\mathscr{E}^{n}=Q \mathscr{E}^{n-1}
$$

where $\mathscr{E}^{n}=\widetilde{U}^{n}-U^{n}$ and $Q=(I+H)^{-1}(I-H)$.

By (82), we have

$$
\mathscr{E}^{n}=Q^{n-1} \mathscr{E}^{0}
$$

Using (83) and Lemma 5, one has

$$
\begin{aligned}
\left\|\mathscr{E}^{n}\right\|_{\infty} & \leq\left\|Q^{n-1}\right\|_{\infty}\left\|\mathscr{E}^{0}\right\|_{\infty} \\
& \leq\|Q\|_{\infty}^{n-1}\left\|\mathscr{E}^{0}\right\|_{\infty} \leq\left\|\mathscr{E}^{0}\right\|_{\infty},
\end{aligned}
$$

which means difference scheme (71) is unconditionally stable, and so is the difference scheme (69).

\section{Convergence Analysis}

In this section, we give the local truncation error analysis of difference scheme (69).

Theorem 10. The local truncation error of numerical scheme (69) for approximating the space Riesz fractional diffusion equation (1) at the point $\left(x_{m}, t_{n}\right)$ is $\mathcal{O}\left(\tau^{2}+h^{4}\right)$.
Proof. We define the local truncation error at the point $\left(x_{m}, t_{n}\right)$ as

$$
\mathscr{R}_{m}^{n}=\frac{2\left[u\left(x_{m}, t_{n}\right)-u\left(x_{m}, t_{n-1}\right)\right]}{\tau}
$$

$$
\begin{aligned}
& +\frac{2}{\tau}\left[\mu_{2} \sum_{k=-M+m+1}^{m-1} g_{k}^{(\alpha)} u\left(x_{m-k}, t_{n}\right)\right. \\
& -\mu_{1} \sum_{k=-M+m+1}^{m-1} g_{k}^{(\alpha)} u\left(x_{m-k-1}, t_{n}\right) \\
& \left.-\mu_{1} \sum_{k=-M+m+1}^{m-1} g_{k}^{(\alpha)} u\left(x_{m-k+1}, t_{n}\right)\right] \\
& +\frac{2}{\tau}\left[\mu_{2} \sum_{k=-M+m+1}^{m-1} g_{k}^{(\alpha)} u\left(x_{m-k}, t_{n-1}\right)\right. \\
& -\mu_{1} \sum_{k=-M+m+1}^{m-1} g_{k}^{(\alpha)} u\left(x_{m-k-1}, t_{n-1}\right) \\
& \left.-\mu_{1} \sum_{k=-M+m+1}^{m-1} g_{k}^{(\alpha)} u\left(x_{m-k+1}, t_{n-1}\right)\right] \\
& -f\left(x_{m}, t_{n}\right)-f\left(x_{m}, t_{n-1}\right) \text {. }
\end{aligned}
$$

Utilizing the Taylor expansion, we have

$$
\begin{aligned}
& \frac{2\left[u\left(x_{m}, t_{n}\right)-u\left(x_{m}, t_{n-1}\right)\right]}{\tau} \\
& =\frac{\partial u\left(x_{m}, t_{n}\right)}{\partial t}+\frac{\partial u\left(x_{m}, t_{n-1}\right)}{\partial t}+\mathcal{O}\left(\tau^{2}\right) .
\end{aligned}
$$

Based on (63) and (86), we obtain

$$
\begin{gathered}
\mathscr{R}_{m}^{n}=\left[\frac{\partial u\left(x_{m}, t_{n}\right)}{\partial t}-K \frac{\partial^{\alpha} u\left(x_{m}, t_{n}\right)}{\partial|x|^{\alpha}}-f\left(x_{m}, t_{n}\right)\right] \\
+\left[\frac{\partial u\left(x_{m}, t_{n-1}\right)}{\partial t}-K \frac{\partial^{\alpha} u\left(x_{m}, t_{n}\right)}{\partial|x|^{\alpha}}\right. \\
\left.-f\left(x_{m}, t_{n}\right)\right]+\mathcal{O}\left(\tau^{2}+h^{4}\right) .
\end{gathered}
$$

Substituting (1) into (87), we get the following local truncation error:

$$
\mathscr{R}_{m}^{n}=\mathcal{O}\left(\tau^{2}+h^{4}\right) \text {. }
$$

Next, we study the convergence of difference scheme (69).

Theorem 11. The order of the convergence of difference scheme (69) is $\mathcal{O}\left(\tau^{2}+h^{4}\right)$; that is to say, there exists a positive constant $C$ such that

$$
\left|u\left(x_{m}, t_{k}\right)-u_{m}^{k}\right| \leq C\left(\tau^{2}+h^{4}\right) .
$$


Proof. Let $e_{m}^{n}=u\left(x_{m}, t_{k}\right)-u_{m}^{k}$. Then it follows from (69) and (85) that

$$
\begin{aligned}
e_{m}^{n}- & \mu_{1} \sum_{k=-M+m+1}^{m-1} g_{k} e_{m-k-1}^{n} \\
& -\mu_{1} \sum_{k=-M+m+1}^{m-1} g_{k} e_{m-k+1}^{n} \\
& +\mu_{2} \sum_{k=-M+m+1}^{m-1} g_{k} e_{m-k}^{n} \\
= & e_{m}^{n-1}+\mu_{1} \sum_{k=-M+m+1}^{m-1} g_{k} e_{m-k-1}^{n-1} \\
& +\mu_{1} \sum_{k=-M+m+1}^{m-1} g_{k} e_{m-k+1}^{n-1} \\
& -\mu_{2} \sum_{k=-M+m+1}^{m-1} g_{k} e_{m-k}^{n-1}+\frac{\tau}{2} \mathscr{R}_{m}^{n} .
\end{aligned}
$$

Denote

$$
E^{n}=\left(e_{1}^{n}, e_{2}^{n}, \ldots, e_{M-1}^{n}\right)^{T}, \quad R^{n}=\left(\mathscr{R}_{1}^{n}, \mathscr{R}_{2}^{n}, \ldots, \mathscr{R}_{M-1}^{n}\right)^{T} ;
$$

then (90) can be written in a matrix form

$$
(I+H) E^{n}=(I-H) E^{n-1}+\frac{\tau}{2} R^{n} .
$$

Furthermore, we can obtain

$$
\begin{aligned}
E^{n} & =Q E^{n-1}+S \\
& =Q\left(Q E^{n-2}+S\right)+S \\
& =\cdots \\
& =\left(Q^{n-1}+Q^{n-2}+\cdots+I\right) S,
\end{aligned}
$$

where $S=(\tau / 2)(I+H)^{-1} R^{n}$.

According to Lemma 5 and Theorem 10, we get

$$
\begin{aligned}
\|S\|_{\infty} & =\left\|\frac{\tau}{2}(I+H)^{-1} R^{n}\right\|_{\infty} \\
& \leq \frac{\tau}{2}\left\|(I+H)^{-1}\right\|_{\infty}\left\|R^{n}\right\|_{\infty} \\
& \leq \tau C_{1}\left(\tau^{2}+h^{4}\right) .
\end{aligned}
$$

Combining (93) and (94) gives

$$
\begin{aligned}
\left\|E^{n}\right\|_{\infty} & =\left\|\left(Q^{n-1}+Q^{n-2}+\cdots+I\right) S\right\|_{\infty} \\
& \leq\left\|\left(Q^{n-1}+Q^{n-2}+\cdots+I\right)\right\|_{\infty}\|S\|_{\infty} \\
& \leq\left(\left\|Q^{n-1}\right\|_{\infty}+\left\|Q^{n-2}\right\|_{\infty}+\cdots+\|I\|_{\infty}\right)\|S\|_{\infty} \\
& \leq\left(\|Q\|_{\infty}^{n-1}+\|Q\|_{\infty}^{n-2}+\cdots+\|I\|_{\infty}\right)\|S\|_{\infty} \\
& \leq n \tau C_{1}\left(\tau^{2}+h^{4}\right) .
\end{aligned}
$$

Noting that $n \tau \leq N \tau=T$, we easily get

$$
\left\|E^{n}\right\|_{\infty} \leq T C_{1}\left(\tau^{2}+h^{4}\right)=C\left(\tau^{2}+h^{4}\right),
$$

where $C=T C_{1}$. This completes the proof.

\section{Numerical Examples}

In the present section, some numerical examples are presented to demonstrate the theoretical analysis.

Example 1. Consider the function $u(x)=x^{2}(1-x)^{2}, x \in$ $[0,1]$.

The Riesz fractional derivative of the function $u(x)$ is

$$
\begin{aligned}
\frac{\partial^{\alpha} u(x)}{\partial|x|^{\alpha}}=-\sec \left(\frac{\pi}{2} \alpha\right)\{ & \frac{1}{\Gamma(3-\alpha)}\left[x^{2-\alpha}+(1-x)^{2-\alpha}\right] \\
& -\frac{6}{\Gamma(4-\alpha)}\left[x^{3-\alpha}+(1-x)^{3-\alpha}\right] \\
& \left.+\frac{12}{\Gamma(5-\alpha)}\left[x^{4-\alpha}+(1-x)^{4-\alpha}\right]\right\} .
\end{aligned}
$$

Table 1 lists the absolute errors and convergence orders at $x=0.5$ by numerical scheme (46) for function $u(x)$ with different $\alpha$.

Example 2. Consider the function $u(x)=x^{6}(1-x)^{6}, x \in$ $[0,1]$.

The Riesz fractional derivative of the function $u(x)$ is

$$
\begin{aligned}
& \frac{\partial^{\alpha} u(x)}{\partial|x|^{\alpha}}=\frac{1}{2} e^{t} \sec \left(\frac{\pi}{2} \alpha\right)\left\{\frac{\Gamma(7)}{\Gamma(7-\alpha)}\left[x^{6-\alpha}+(1-x)^{6-\alpha}\right]\right. \\
& -\frac{6 \Gamma(8)}{\Gamma(8-\alpha)}\left[x^{7-\alpha}+(1-x)^{7-\alpha}\right] \\
& +\frac{15 \Gamma(9)}{\Gamma(9-\alpha)}\left[x^{8-\alpha}+(1-x)^{8-\alpha}\right] \\
& -\frac{20 \Gamma(10)}{\Gamma(10-\alpha)}\left[x^{9-\alpha}+(1-x)^{9-\alpha}\right] \\
& +\frac{15 \Gamma(8)}{\Gamma(11-\alpha)}\left[x^{10-\alpha}+(1-x)^{10-\alpha}\right] \\
& -\frac{6 \Gamma(12)}{\Gamma(12-\alpha)}\left[x^{11-\alpha}+(1-x)^{11-\alpha}\right] \\
& \left.+\frac{\Gamma(13)}{\Gamma(13-\alpha)}\left[x^{12-\alpha}+(1-x)^{12-\alpha}\right]\right\} \text {. }
\end{aligned}
$$

Table 2 lists the absolute errors and convergence orders at $x=0.5$ by numerical scheme (47) for function $u(x)$ with different $\alpha$. These computational results confirm the secondorder and fourth-order of the numerical formulas (46) and (47), respectively. 
TABLE 1: The absolute errors and convergence orders of Example 1 by numerical scheme (46).

\begin{tabular}{|c|c|c|c|}
\hline$\alpha$ & $h$ & The absolute error & The convergence order \\
\hline \multirow{4}{*}{0.2} & $\frac{1}{10}$ & $3.630966 e-003$ & - \\
\hline & $\frac{1}{20}$ & $9.120270 e-004$ & 1.9932 \\
\hline & $\frac{1}{40}$ & $2.285315 e-004$ & 1.9967 \\
\hline & $\frac{1}{80}$ & $5.719787 e-005$ & 1.9984 \\
\hline \multirow{4}{*}{0.4} & $\frac{1}{10}$ & $5.124542 e-003$ & - \\
\hline & $\frac{1}{20}$ & $1.289681 e-003$ & 1.9904 \\
\hline & $\frac{1}{40}$ & $3.234889 e-004$ & 1.9952 \\
\hline & $\frac{1}{80}$ & $8.100606 e-005$ & 1.9976 \\
\hline \multirow{4}{*}{0.6} & $\frac{1}{10}$ & $4.629914 e-003$ & - \\
\hline & $\frac{1}{20}$ & $1.164707 e-003$ & 1.9910 \\
\hline & $\frac{1}{40}$ & $2.920982 e-004$ & 1.9954 \\
\hline & $\frac{1}{80}$ & $7.314118 e-005$ & 1.9977 \\
\hline \multirow{4}{*}{0.8} & $\frac{1}{10}$ & $2.652282 e-003$ & - \\
\hline & $\frac{1}{20}$ & $6.653815 e-004$ & 1.9950 \\
\hline & $\frac{1}{40}$ & $1.666617 e-004$ & 1.9973 \\
\hline & $\frac{1}{80}$ & $4.170691 e-005$ & 1.9986 \\
\hline \multirow{4}{*}{1.2} & $\frac{1}{10}$ & $2.371107 e-003$ & - \\
\hline & $\frac{1}{20}$ & $5.878877 e-004$ & 2.0119 \\
\hline & $\frac{1}{40}$ & $1.464631 e-004$ & 2.0050 \\
\hline & $\frac{1}{80}$ & $3.655831 e-005$ & 2.0023 \\
\hline \multirow{4}{*}{1.4} & $\frac{1}{10}$ & $3.614913 e-003$ & - \\
\hline & $\frac{1}{20}$ & $8.891952 e-004$ & 2.0234 \\
\hline & $\frac{1}{40}$ & $2.207491 e-004$ & 2.0101 \\
\hline & $\frac{1}{80}$ & $5.500869 e-005$ & 2.0047 \\
\hline
\end{tabular}

TABLE 1: Continued.

\begin{tabular}{cccc}
\hline$\alpha$ & $h$ & The absolute error & The convergence order \\
\hline & $\frac{1}{10}$ & $3.311900 e-003$ & - \\
& $\frac{1}{20}$ & $8.088550 e-004$ & 2.0337 \\
& $\frac{1}{40}$ & $2.001866 e-004$ & 2.0145 \\
$\frac{1}{80}$ & $4.981273 e-005$ & 2.0068 \\
\hline$\frac{1}{10}$ & $1.748268 e-003$ & - \\
$\frac{1}{20}$ & $4.265789 e-004$ & 2.0350 \\
$\frac{1}{40}$ & $1.055454 e-004$ & 2.0149 \\
$\frac{1}{80}$ & $2.625954 e-005$ & 2.0069 \\
\hline
\end{tabular}

Example 3. We consider the equation

$$
\begin{array}{r}
\frac{\partial u(x, t)}{\partial t}=K \frac{\partial^{\alpha} u(x, t)}{\partial|x|^{\alpha}}+f(x, t), \\
0<x<1, \quad 0<t \leq 1,
\end{array}
$$

where

$$
\begin{aligned}
f(x, t)=(2 \alpha+1) t^{2 \alpha} x^{4}(1-x)^{4}+t^{2 \alpha+1} \sec \left(\frac{\pi}{2} \alpha\right) \\
\times\left\{\frac{12}{\Gamma(5-\alpha)}\left[x^{4-\alpha}+(1-x)^{4-\alpha}\right]\right. \\
\quad-\frac{240}{\Gamma(6-\alpha)}\left[x^{5-\alpha}+(1-x)^{5-\alpha}\right] \\
+\frac{2160}{\Gamma(7-\alpha)}\left[x^{6-\alpha}+(1-x)^{6-\alpha}\right] \\
\quad-\frac{10080}{\Gamma(8-\alpha)}\left[x^{7-\alpha}+(1-x)^{7-\alpha}\right] \\
\left.+\frac{20160}{\Gamma(9-\alpha)}\left[x^{8-\alpha}+(1-x)^{8-\alpha}\right]\right\}
\end{aligned}
$$

together with the initial condition $u(x, 0)=0$ and homogeneous boundary value conditions.

The analytical solution of (99) is $u(x, t)=t^{2 \alpha+1} x^{4}(1-x)^{4}$.

Table 3 lists the maximum error and time and space convergence orders for different $\alpha$. Numerical results show that the convergence order of the difference scheme (69) is $O\left(\tau^{2}+h^{4}\right)$, which are in line with our theoretical analysis.

Figures 1 and 2 show the comparison of the analytical and numerical solutions with $\alpha=1.1$ at $t=0.2$ and $\alpha=1.9$ at $t=$ 0.8 , respectively. It can be seen that the numerical solutions are in excellent agreement with the analytical solutions. 
TABLE 2: The absolute errors and convergence orders of Example 2 by numerical scheme (47)

\begin{tabular}{|c|c|c|c|}
\hline$\alpha$ & $h$ & The absolute error & The convergence order \\
\hline \multirow{4}{*}{0.2} & $\frac{1}{20}$ & $1.571776 e-008$ & - \\
\hline & $\frac{1}{40}$ & $9.923695 e-010$ & 3.9854 \\
\hline & $\frac{1}{80}$ & $6.218021 e-011$ & 3.9963 \\
\hline & $\frac{1}{160}$ & $3.888658 e-012$ & 3.9991 \\
\hline \multirow{4}{*}{0.4} & $\frac{1}{20}$ & $6.255711 e-008$ & - \\
\hline & $\frac{1}{40}$ & $3.955450 e-009$ & 3.9833 \\
\hline & $\frac{1}{80}$ & $2.479333 e-010$ & 3.9958 \\
\hline & $\frac{1}{160}$ & $1.550707 e-011$ & 3.9990 \\
\hline \multirow{4}{*}{0.6} & $\frac{1}{20}$ & $1.660570 e-007$ & - \\
\hline & $\frac{1}{40}$ & $1.051535 e-008$ & 3.9811 \\
\hline & $\frac{1}{80}$ & $6.593628 e-010$ & 3.9953 \\
\hline & $\frac{1}{160}$ & $4.124394 e-011$ & 3.9988 \\
\hline \multirow{4}{*}{0.8} & $\frac{1}{20}$ & $3.501076 e-007$ & - \\
\hline & $\frac{1}{40}$ & $2.219986 e-008$ & 3.9792 \\
\hline & $\frac{1}{80}$ & $1.392502 e-009$ & 3.9948 \\
\hline & $\frac{1}{160}$ & $8.711096 e-011$ & 3.9987 \\
\hline \multirow{4}{*}{1.2} & $\frac{1}{20}$ & $7.029869 e-007$ & - \\
\hline & $\frac{1}{40}$ & $4.446255 e-008$ & 3.9828 \\
\hline & $\frac{1}{80}$ & $2.787163 e-009$ & 3.9957 \\
\hline & $\frac{1}{160}$ & $1.743236 e-010$ & 3.9990 \\
\hline \multirow{4}{*}{1.4} & $\frac{1}{20}$ & $4.372243 e-008$ & - \\
\hline & $\frac{1}{40}$ & $3.985260 e-009$ & 3.4556 \\
\hline & $\frac{1}{80}$ & $2.690303 e-010$ & 3.8888 \\
\hline & $\frac{1}{160}$ & $1.712778 e-011$ & 3.9734 \\
\hline
\end{tabular}

TABLE 2: Continued.

\begin{tabular}{cccc}
$\alpha$ & $h$ & The absolute error & The convergence order \\
\hline & $\frac{1}{20}$ & $3.751536 e-006$ & - \\
1.6 & $\frac{1}{40}$ & $2.440706 e-007$ & 3.9421 \\
$\frac{1}{80}$ & $1.540687 e-008$ & 3.9857 \\
$\frac{1}{160}$ & $9.653215 e-010$ & 3.9964 \\
$\frac{1}{20}$ & $1.592788 e-005$ & - \\
$\frac{1}{40}$ & $1.034107 e-006$ & 3.9451 \\
$\frac{1}{80}$ & $6.524548 e-008$ & 3.9864 \\
$\frac{1}{160}$ & $4.087469 e-009$ & 3.9966 \\
\hline
\end{tabular}

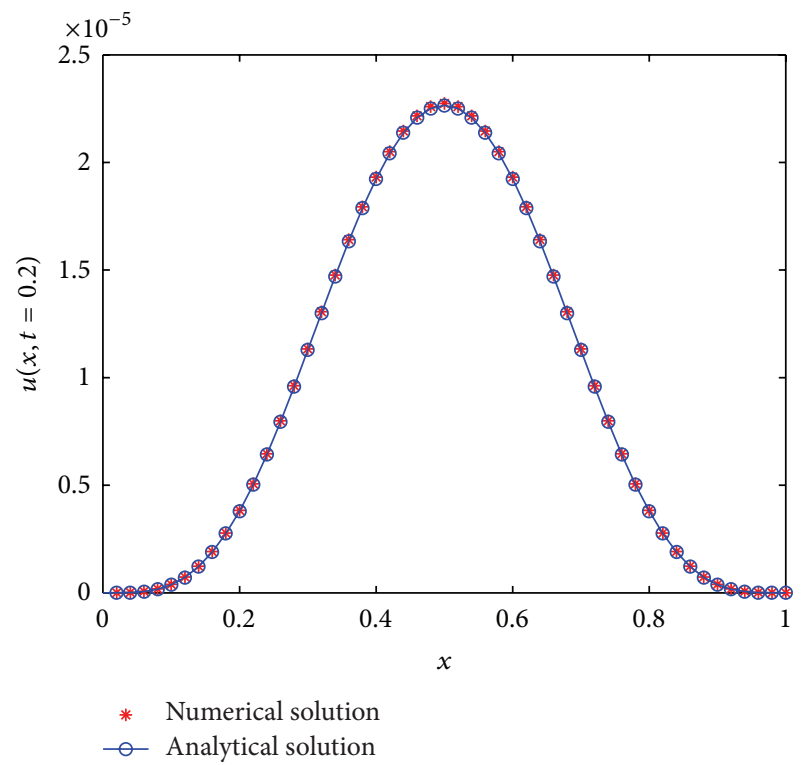

Figure 1: Comparison between the analytical solution and the numerical solution at $t=0.2$ with $\alpha=1.1$ in Example $3(\tau=$ $1 / 50, h=1 / 50)$.

Figures 3, 4, 5, and 6 display the numerical and analytical solutions surface with $\alpha=1.2$ and $\alpha=1.8$. By Figures 3-6, we find that the corresponding two groups of figures are almost the same, respectively.

\section{Conclusions}

In this paper, we construct one second-order method and two fourth-order methods for the Riesz fractional derivative. 
TABLE 3: The maximum errors and temporal and spatial convergence orders of Example 3 by difference scheme (66).

\begin{tabular}{|c|c|c|c|c|}
\hline$\alpha$ & & The maximum errors & Temporal convergence orders & Spatial convergence orders \\
\hline \multirow{10}{*}{1.2} & $h=\frac{1}{4}, \tau=\frac{1}{2}$ & $3.070974 e-004$ & - & - \\
\hline & $h=\frac{1}{0}, \tau=\frac{1}{0}$ & $2038855 e-005$ & 19564 & 3.9129 \\
\hline & $\begin{array}{ll}8 & 8 \\
1 & 1\end{array}$ & $2.058855 e-005$ & 1.9504 & 3.9129 \\
\hline & $h=\frac{1}{16}, \tau=\frac{1}{32}$ & $1.318397 e-006$ & 1.9755 & 3.9509 \\
\hline & $h-1 \tau-1$ & & & \\
\hline & $h=\overline{32}, \tau=\overline{128}$ & $8.371766 e-008$ & 1.9886 & 3.9771 \\
\hline & $h=\frac{1}{,} \tau=1$ & & & \\
\hline & $-\overline{64},-\overline{512}$ & $5.279883 e-009$ & 1.9935 & 3.9870 \\
\hline & $h=\frac{1}{s}, \tau=\frac{1}{2}$ & & 19603 & \\
\hline & $128 \quad 2048$ & $3.480 / 07 e^{-010}$ & 1.9003 & 3.9206 \\
\hline \multirow{10}{*}{1.4} & $h=\frac{1}{4}, \tau=\frac{1}{2}$ & $3.715656 e-004$ & - & - \\
\hline & $h=\frac{1}{,} \tau=\frac{1}{-}$ & & & \\
\hline & $n-\overline{8}, \iota-\overline{8}$ & $2.465865 e-005$ & 1.9567 & 3.9135 \\
\hline & $h=\frac{1}{11}, \tau=\frac{1}{22}$ & $1603083 e-006$ & 10716 & 30432 \\
\hline & $\begin{array}{ll}16 & 32\end{array}$ & & 1. & 3.9432 \\
\hline & $h=\frac{1}{32}, \tau=\frac{1}{128}$ & $1.022389 e-007$ & 19854 & 39708 \\
\hline & $\begin{array}{cc}52 & 120 \\
1 & 1\end{array}$ & & & 0.9700 \\
\hline & $h=\frac{\overline{6}}{64}, \tau=\frac{\overline{512}}{512}$ & $6.472320 e-009$ & 1.9908 & 3.9815 \\
\hline & $h=\frac{1}{}, \tau=\frac{1}{}$ & & & \\
\hline & $n=\overline{128}, \tau=\overline{2048}$ & $4.083361 e-010$ & 1.9932 & 3.9865 \\
\hline \multirow{10}{*}{1.6} & $h=\frac{1}{4}, \tau=\frac{1}{2}$ & $4.193160 e-004$ & - & - \\
\hline & $h=\frac{1}{,}, \tau=\frac{1}{3}$ & $27753950-005$ & 10586 & 30173 \\
\hline & $\begin{array}{cc}8 & 8 \\
1 & \end{array}$ & $2.1750900-005$ & 1.95000 & $3.91 / 3$ \\
\hline & $h=\frac{1}{16}, \tau=\frac{1}{32}$ & $1810618 e-006$ & 19691 & 39381 \\
\hline & $\begin{array}{lc}16 & 32 \\
1 & 1\end{array}$ & 1.07000000 & ד & 0.9501 \\
\hline & $h=\frac{1}{32}, \tau=\frac{1}{128}$ & $1158839 e-007$ & 19829 & 39657 \\
\hline & $\begin{array}{cc}32 & 128 \\
1 & 1\end{array}$ & (1) & 1.0028 & (5) \\
\hline & $h=\overline{64}, \tau=\overline{512}$ & $7.363046 e-009$ & 1.9881 & 3.9762 \\
\hline & $h=\frac{1}{}, \tau=\frac{1}{2}$ & & & \\
\hline & $n=\overline{128}, \tau=\overline{2048}$ & $4.662698 e-010$ & 1.9905 & 3.9811 \\
\hline \multirow{10}{*}{1.8} & $h=\frac{1}{4}, \tau=\frac{1}{2}$ & $4.417615 e-004$ & - & - \\
\hline & $h=\frac{1}{\rho}, \tau=\frac{1}{\rho}$ & $2.895409 e-005$ & 1.9657 & 3.9314 \\
\hline & $\begin{array}{cc}8 & 8 \\
1 & 1\end{array}$ & - & & \\
\hline & $h=\frac{\tau}{16}, \tau=\frac{\overline{32}}{32}$ & $1.884230 e-006$ & 1.9709 & 3.9417 \\
\hline & $1-1$ & & & \\
\hline & $h=\overline{32}, \tau=\overline{128}$ & $1.204909 e-007$ & 1.9835 & 3.9670 \\
\hline & $h=\frac{1}{}, \tau=1$ & & & \\
\hline & $n-\overline{64}, \iota-\overline{512}$ & $7.658088 e-009$ & 1.9879 & 3.9758 \\
\hline & $h=\frac{1}{\tau}, \tau=\frac{1}{2}$ & $4.855499 e-010$ & 1.9896 & 39793 \\
\hline & $128 \quad 2048$ & מים & & 0.9190 \\
\hline
\end{tabular}

Later on, a fourth-order numerical formula is used for the Riesz space fractional diffusion equation. The stability and convergence of this numerical method are analyzed by the matrix method in detail. Finally, some numerical results are given to demonstrate the effectiveness of numerical method. From the above results, it is possible to claim that the methods and techniques discussed in this paper are useful for solving some other fractional differential equations with Riesz derivatives.

\section{Conflict of Interests}

The authors declare that there is no conflict of interests regarding the publication of this paper. 


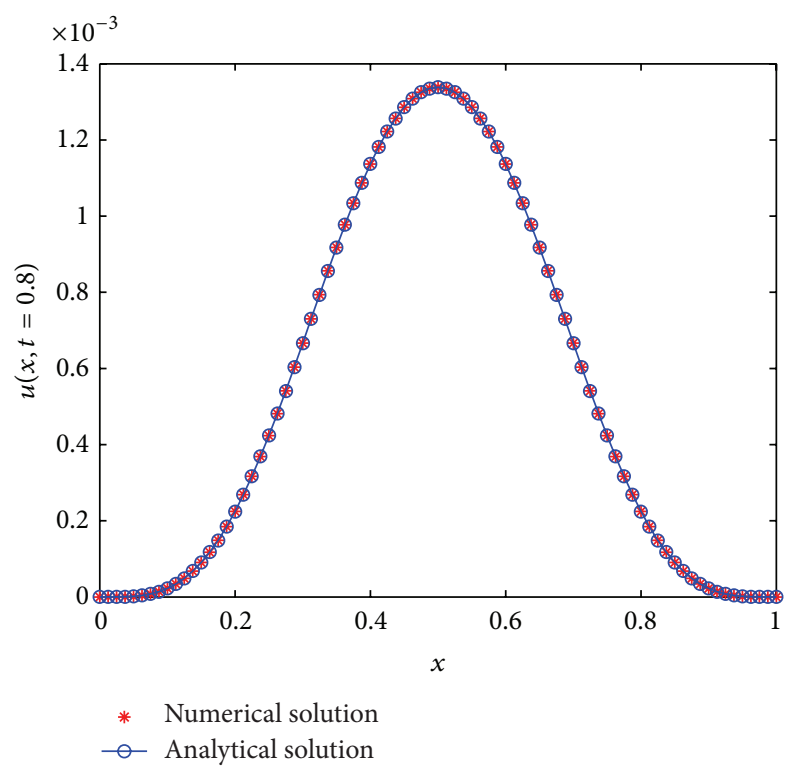

FIgURE 2: Comparison between the analytical solution and the numerical solution at $t=0.8$ with $\alpha=1.9$ in Example $3(\tau=1 / 100$, $h=1 / 80)$.

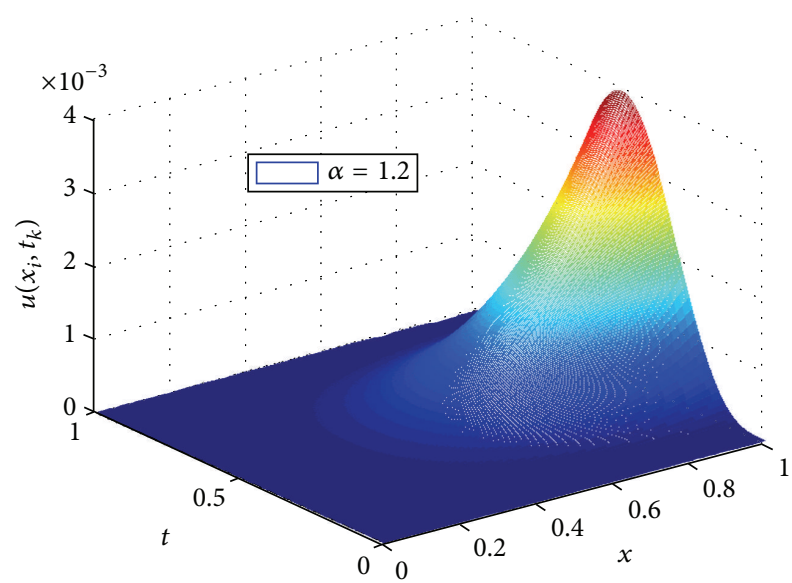

FIgURE 3: The numerical solution surface when $\alpha=1.2$ in Example $3(\tau=1 / 500, h=1 / 100)$.

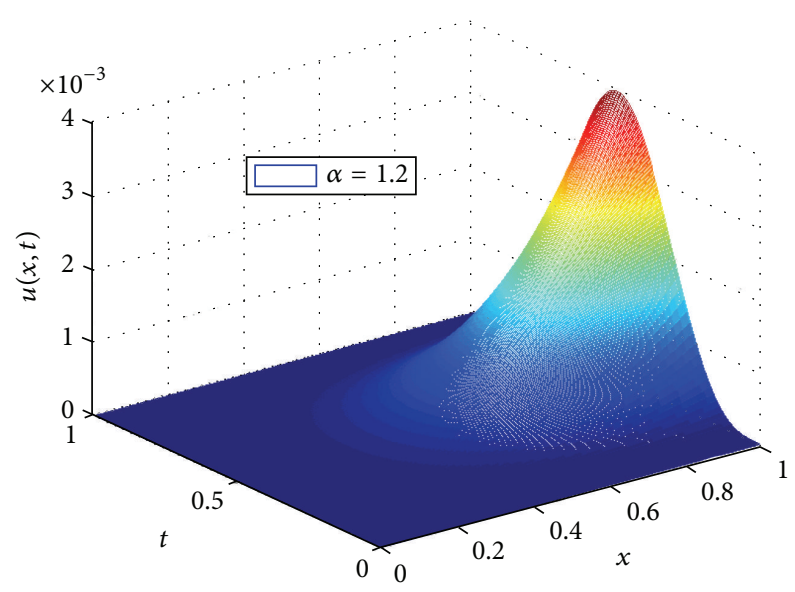

FIgURE 4: The analytical solution surface when $\alpha=1.2$ in Example 3.

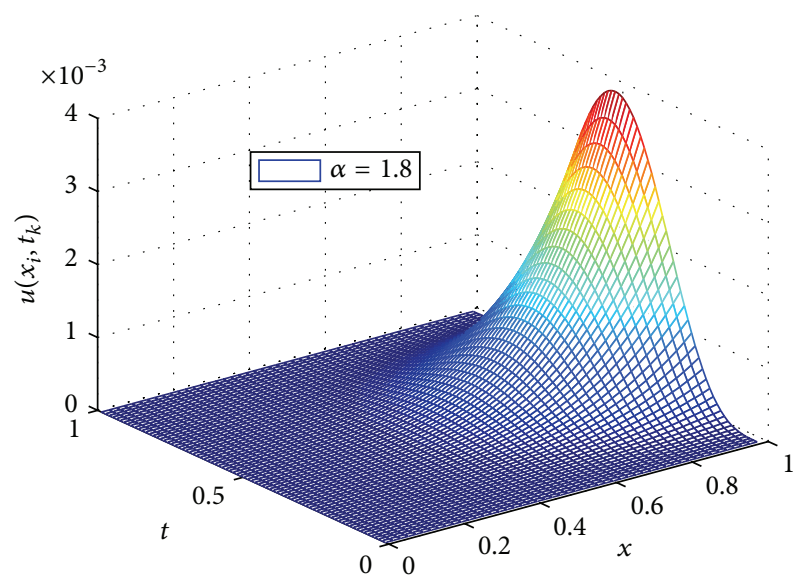

Figure 5: The numerical solution surface when $\alpha=1.8$ in Example $3(\tau=1 / 50, h=1 / 80)$.

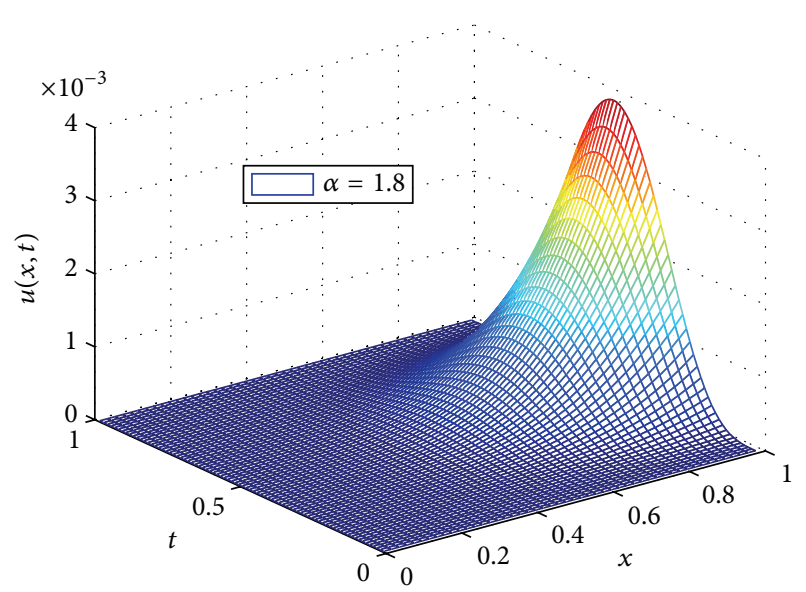

FIGURE 6: The analytical solution surface when $\alpha=1.8$ in Example 3.

\section{Acknowledgment}

This work was partially supported by Key Program of Shanghai Municipal Education Commission under Grant no. 12ZZ084 and the grant of "The First-Class Discipline of Universities in Shanghai."

\section{References}

[1] B. L. Guo, X. K. Pu, and F. H. Huang, Fractional Partial Differential Equations and Their Numerical Solutions, Science Press, Beijing, China, 2011 (Chinese).

[2] A. A. Kilbas, H. M. Srivastava, and J. J. Trujillo, Theory and Applications of Fractional Differential Equations, vol. 204 of NorthHolland Mathematics Studies, Elsevier Science, Amsterdam, The Netherlands, 2006.

[3] K. B. Oldham and J. Spanier, The Fractional Calculus: Theory and Applications of Differentiation and Integration to Arbitrary Order, Academic Press, New York, NY, USA, 1974.

[4] J. Chen, F. Liu, and V. Anh, "Analytical solution for the timefractional telegraph equation by the method of separating variables," Journal of Mathematical Analysis and Applications, vol. 338, no. 2, pp. 1364-1377, 2008. 
[5] F. Huang and B. Guo, "General solutions to a class of time fractional partial differential equations," Applied Mathematics and Mechanics, vol. 31, no. 7, pp. 815-826, 2010.

[6] I. Podlubny, Fractional Differential Equations, vol. 198 of Mathematics in Science and Engineering, Academic Press, San Diego, Calif, USA, 1999.

[7] N. T. Shawagfeh, "Analytical approximate solutions for nonlinear fractional differential equations," Applied Mathematics and Computation, vol. 131, no. 2-3, pp. 517-529, 2002.

[8] Ch. Lubich, "Discretized fractional calculus," SIAM Journal on Mathematical Analysis, vol. 17, no. 3, pp. 704-719, 1986.

[9] C. Li, A. Chen, and J. Ye, "Numerical approaches to fractional calculus and fractional ordinary differential equation," Journal of Computational Physics, vol. 230, no. 9, pp. 3352-3368, 2011.

[10] E. Hanert, "On the numerical solution of space-time fractional diffusion models," Computers \& Fluids, vol. 46, pp. 33-39, 2011.

[11] W. McLean and K. Mustapha, "A second-order accurate numerical method for a fractional wave equation," Numerische Mathematik, vol. 105, no. 3, pp. 481-510, 2007.

[12] K. Mustapha and W. McLean, "Superconvergence of a discontinuous Galerkin method for fractional diffusion and wave equations," SIAM Journal on Numerical Analysis, vol. 51, no. 1, pp. 491-515, 2013.

[13] C. Piret and E. Hanert, "A radial basis functions method for fractional diffusion equations," Journal of Computational Physics, vol. 238, pp. 71-81, 2013.

[14] A. I. Saichev and G. M. Zaslavsky, "Fractional kinetic equations: solutions and applications," Chaos, vol. 7, no. 4, pp. 753-764, 1997.

[15] G. M. Zaslavsky, "Chaos, fractional kinetics, and anomalous transport," Physics Reports, vol. 371, no. 6, pp. 461-580, 2002.

[16] H. Zhang and F. Liu, "The fundamental solutions of the space, space-time Riesz fractional partial differential equations with periodic conditions," Numerical Mathematics: A Journal of Chinese Universities, English Series, vol. 16, no. 2, pp. 181-192, 2007.

[17] H. Zhang, F. Liu, and V. Anh, "Galerkin finite element approximation of symmetric space-fractional partial differential equations," Applied Mathematics and Computation, vol. 217, no. 6, pp. 2534-2545, 2010.

[18] J. Chen, F. Liu, I. Turner, and V. Anh, “The fundamental and numerical solutions of the Riesz space-fractional reactiondispersion equation," The ANZIAM Journal, vol. 50, no. 1, pp. 45-57, 2008.

[19] S. Shen, F. Liu, and V. Anh, "Numerical approximations and solution techniques for the space-time Riesz-Caputo fractional advection-diffusion equation," Numerical Algorithms, vol. 56, no. 3, pp. 383-403, 2011.

[20] S. Shen, F. Liu, V. Anh, and I. Turner, "A novel numerical approximation for the space fractional advection-dispersion equation," IMA Journal of Applied Mathematics, 2012.

[21] N. Özdemir, D. Avc1, and B. B. İskender, "The numerical solutions of a two-dimensional space-time Riesz-Caputo fractional diffusion equation," International Journal of Optimization and Control: Theories \& Applications, vol. 1, no. 1, pp. 17-26, 2011.

[22] Q. Yang, F. Liu, and I. Turner, "Numerical methods for fractional partial differential equations with Riesz space fractional derivatives," Applied Mathematical Modelling, vol. 34, no. 1, pp. 200-218, 2010.

[23] C. Celik and M. Duman, "Crank-Nicolson method for the fractional diffusion equation with the Riesz fractional derivative,"
Journal of Computational Physics, vol. 231, no. 4, pp. 1743-1750, 2012.

[24] D. Wang, A. Xiao, and W. Yang, "Crank-Nicolson difference scheme for the coupled nonlinear Schrödinger equations with the Riesz space fractional derivative," Journal of Computational Physics, vol. 242, pp. 670-681, 2013.

[25] M. M. Meerschaert and C. Tadjeran, "Finite difference approximations for fractional advection-dispersion flow equations," Journal of Computational and Applied Mathematics, vol. 172, no. 1, pp. 65-77, 2004.

[26] E. Sousa, "A second order explicit finite difference method for the fractional advection diffusion equation," Computers \& Mathematics with Applications, vol. 64, no. 10, pp. 3141-3152, 2012.

[27] M. D. Ortigueira, "Riesz potential operators and inverses via fractional centred derivatives," International Journal of Mathematics and Mathematical Sciences, vol. 2006, Article ID 48391, 12 pages, 2006.

[28] W. Y. Tian, H. Zhou, and W. H. Deng, "A class of secondorder difference approximation for solving space fractional diffusion equations," http://arxiv.org/abs/1201.5949.

[29] V. J. Ervin and J. P. Roop, "Variational formulation for the stationary fractional advection dispersion equation," Numerical Methods for Partial Differential Equations, vol. 22, no. 3, pp. 558576, 2006.

[30] V. K. Tuan and R. Gorenflo, "Extrapolation to the limit for numerical fractional differentiation," Zeitschrift für Angewandte Mathematik und Mechanik, vol. 75, no. 8, pp. 646-648, 1995.

[31] W. Zhang, Finite Difference Methods for Partial Differential Equations in Science Computation, Higher Education Press, Beijing, China, 2006 (Chinese).

[32] R. H.-F. Chan and X.-Q. Jin, An Introduction to Iterative Toeplitz Solvers, vol. 5 of Fundamentals of Algorithms, SIAM, Philadelphia, Pa, USA, 2007.

[33] R. H. Chan, "Toeplitz preconditioners for Toeplitz systems with nonnegative generating functions," IMA Journal of Numerical Analysis, vol. 11, no. 3, pp. 333-345, 1991. 


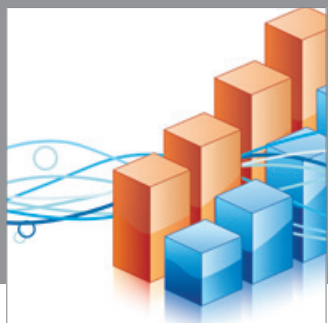

Advances in

Operations Research

mansans

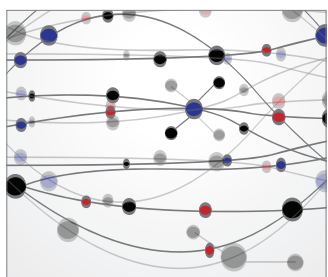

The Scientific World Journal
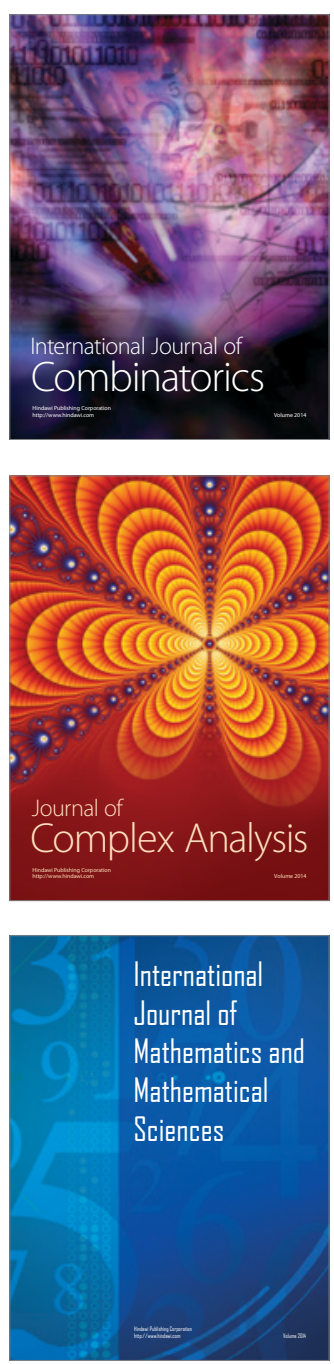
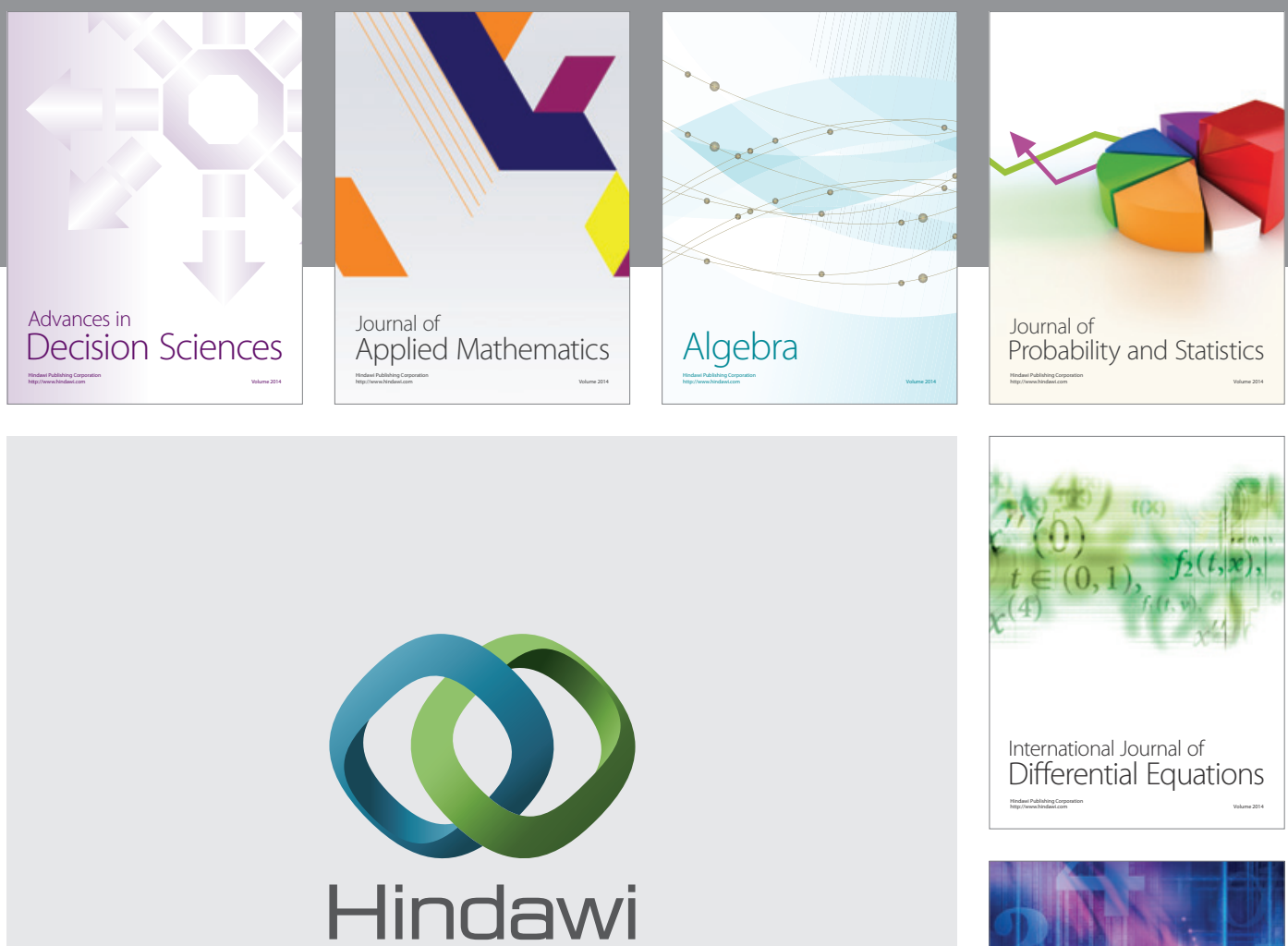

Submit your manuscripts at http://www.hindawi.com
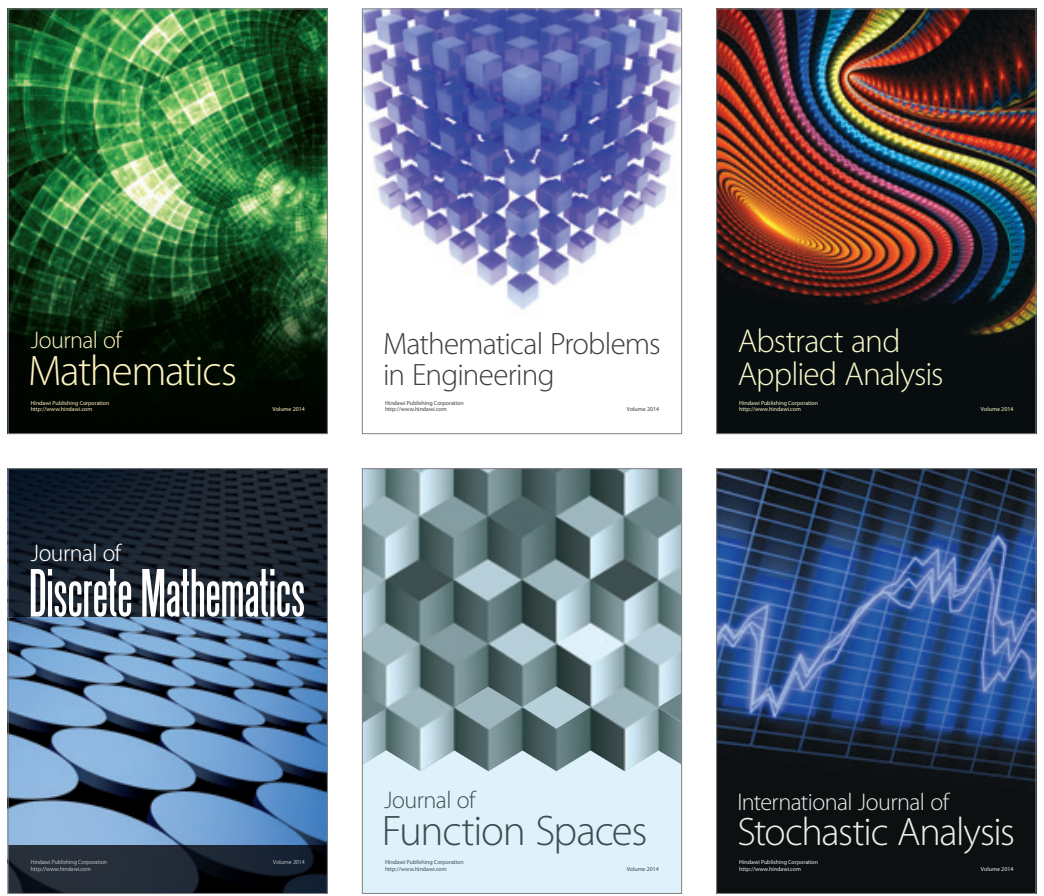

Journal of

Function Spaces

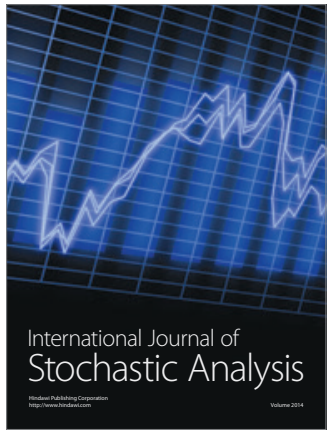

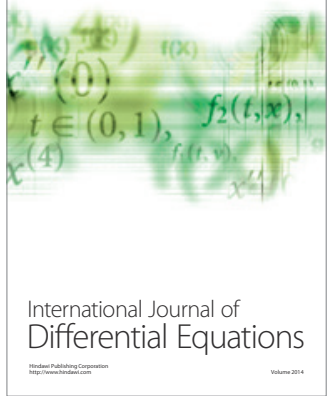
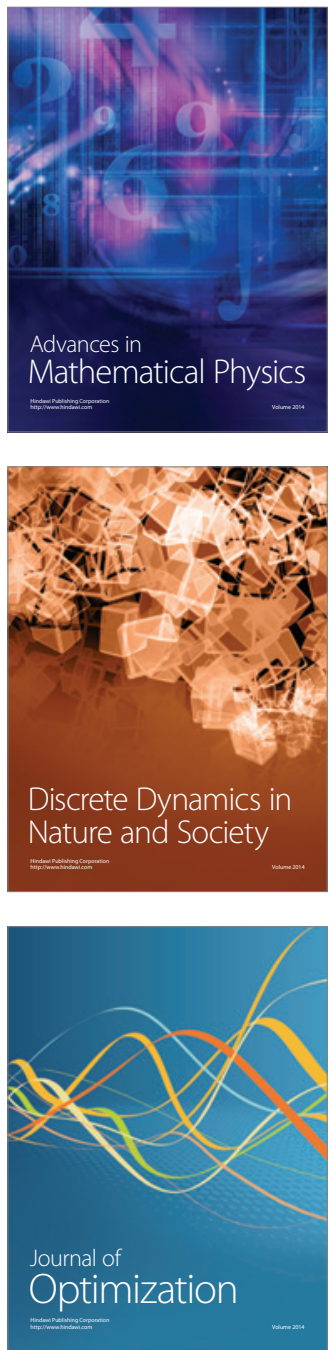